\title{
Matching Energy of Unicyclic and Bicyclic Graphs with a Given Diameter
}

\author{
Lin Chen, Jinfeng Liu, Yongtang Sh] \\ Center for Combinatorics and LPMC-TJKLC, Nankai University, Tianjin \\ 300071, P.R. China \\ E-mails: chenlin1120120012@126.com, ljinfeng709@163.com, shi@nankai.edu.cn
}

\begin{abstract}
Gutman and Wagner proposed the concept of matching energy (ME) and pointed out that the chemical applications of ME go back to the 1970s. Let $G$ be a simple graph of order $n$ and $\mu_{1}, \mu_{2}, \ldots, \mu_{n}$ be the roots of its matching polynomial. The matching energy of $G$ is defined to be the sum of the absolute values of $\mu_{i}(i=1,2, \ldots, n)$. In this paper, we characterize the graphs with minimal matching energy among all unicyclic and bicyclic graphs with a given diameter $d$.
\end{abstract}

Key words: graph; matching energy; energy; diameter

\section{Introduction}

In this paper, all graphs under our consideration are finite, connected, undirected and simple. For more notations and terminology that will be used in the sequel, we refer to 2 . Let $G$ be a simple undirected graph with order $n$ and $A(G)$ be the adjacency matrix of $G$. The characteristic polynomial of $G$, denoted by $\phi(G)$, is defined as

$$
\phi(G)=\operatorname{det}(x I-A(G))=\sum_{i=0}^{n} a_{i}(G) x^{n-i},
$$

where $I$ is the identity matrix of order $n$. The roots of the equation $\phi(G)=$ 0 , denoted by $\lambda_{1}, \lambda_{2}, \ldots, \lambda_{n}$, are the eigenvalues of $A(G)$. The energy of

\footnotetext{
${ }^{1}$ The corresponding author.
} 
$G$, denoted by $E(G)$, is defined as the sum of the absolute values of the eigenvalues of $A(G)$, that is,

$$
E(G)=\sum_{i=1}^{n}\left|\lambda_{i}\right|
$$

The concept of the energy of simple undirected graphs was introduced by Gutman in [15] and now is well-studied. For more results about graph energy, we refer the readers to recent papers [8, 9, 12, 32, 34, two surveys [16, 17] and the book [31. There are various generalizations of graph energy, such as Randić energy [3, 10], Laplacian energy [7, distance energy [36], incidence energy [4, 5], energy of matrices [14] and energy of a polynomial 33, etc.

Let $G$ be a simple graph with $n$ vertices and $m$ edges. Denote by $m_{k}(G)$ the number of $k$-matchings ( $=$ the number of selections of $k$ independent edges $=$ the number of $k$-element independent edge sets) of $G$. Specifically, $m_{1}(G)=m$ and $m_{k}(G)=0$ for $k>\left\lfloor\frac{n}{2}\right\rfloor$ or $k<0$. It is both consistent and convenient to define $m_{0}(G)=1$. The matching polynomial of the graph $G$ is defined as

$$
\alpha(G)=\alpha(G, \mu)=\sum_{k \geq 0}(-1)^{k} m_{k}(G) \mu^{n-2 k} .
$$

Recently, Gutman and Wagner 23] defined the matching energy of a graph $G$ based on the zeros of its matching polynomial [13, 21].

Definition 1.1 Let $G$ be a simple graph with order $n$, and $\mu_{1}, \mu_{2}, \ldots, \mu_{n}$ be the zeros of its matching polynomial. Then,

$$
M E(G)=\sum_{i=1}^{n}\left|\mu_{i}\right|
$$

Moreover, Gutman and Wagner [23] pointed out that the matching energy is a quantity of relevance for chemical applications. They arrived at the simple relation:

$$
\operatorname{TRE}(G)=E(G)-M E(G),
$$

where $\operatorname{TRE}(G)$ is the so-called "topological resonance energy" of $G$. About the chemical applications of matching energy, for more details see [18, 1, 20].

For the coefficients $a_{i}(G)$ of $\phi(G)$, let $b_{i}(G)=\left|a_{i}(G)\right|, i=0,1, \ldots, n$. Note that $b_{0}(G)=1, b_{1}(G)=0$, and $b_{2}(G)$ is the number of edges of $G$. For convenience, let $b_{i}(G)=0$ if $i<0$. In [19, 24, we have

$$
E(G)=\frac{1}{2 \pi} \int_{-\infty}^{\infty} \frac{d x}{x^{2}} \ln \left[\left(\sum_{j=0}^{\left\lfloor\frac{n}{2}\right\rfloor} b_{2 j}(G) x^{2 j}\right)^{2}+\left(\sum_{j=0}^{\left\lfloor\frac{n}{2}\right\rfloor} b_{2 j+1}(G) x^{2 j+1}\right)^{2}\right] .
$$

Thus $E(G)$ is a monotonically increasing function of $b_{i}(G), i=0,1, \ldots, n$. 
Being similar to Eq.(3), the matching energy also has a beautiful formula as follows 23]. Eq.(44) could be considered as the definition of matching energy, in which case Eq.(2) would become a theorem.

Theorem 1.1 Let $G$ be a simple graph of order $n$, and $m_{k}(G)$ be the number of its $k$-matchings, $k=0,1,2, \ldots,\left\lfloor\frac{n}{2}\right\rfloor$. The matching energy of $G$ is given by

$$
M E=M E(G)=\frac{2}{\pi} \int_{0}^{\infty} \frac{1}{x^{2}} \ln \left[\sum_{k \geq 0} m_{k}(G) x^{2 k}\right] d x .
$$

By Eq. (41) and the monotony of the function logarithm, we can define a quasi-order " $\succeq$ " as follows: If two graphs $G_{1}$ and $G_{2}$ have the same order and size, then

$$
G_{1} \succeq G_{2} \Longleftrightarrow m_{k}\left(G_{1}\right) \geq m_{k}\left(G_{2}\right) \text { for all } k \text {. }
$$

If $G_{1} \succeq G_{2}$ and there exists some $k$ such that $m_{k}\left(G_{1}\right)>m_{k}\left(G_{2}\right)$, then we write $G_{1} \succ G_{2}$. Clearly, $G_{1} \succ G_{2} \Longrightarrow M E\left(G_{1}\right)>M E\left(G_{2}\right)$.

Notice that when $M E\left(G_{1}\right)>M E\left(G_{2}\right)$, we may not deduce that $G_{1} \succ$ $G_{2}$. However, if $G$ is any simple connected graph with $n$ vertices other than $S_{n}$, where $S_{n}$ is a star of order $n$, then not only $M E(G)>M E\left(S_{n}\right)$ [23] but also $G \succ S_{n}$. Based on the quasi-order, there are some more extremal results on matching energy of graphs [6, 26, 27, 30].

In this paper, we characterize the graphs with minimal matching energy among all unicyclic and bicyclic graphs with a given diameter $d$.

\section{Preliminaries}

The following result gives two fundamental identities for the number of $k$ matchings of a graph (see [13, 21]).

Lemma 2.1 Let $G$ be a simple graph, $e=u v$ be an edge of $G$, and $N(u)=$ $\left\{v_{1}(=v), v_{2}, \ldots, v_{j}\right\}$ be the set of all neighbors of $u$ in $G$. Then we have

$$
\begin{gathered}
m_{k}(G)=m_{k}(G-u v)+m_{k-1}(G-u-v), \\
m_{k}(G)=m_{k}(G-u)+\sum_{i=1}^{j} m_{k-1}\left(G-u-v_{i}\right) .
\end{gathered}
$$

From Lemma 2.1, we know that $m_{k}\left(P_{1} \cup G\right)=m_{k}(G)$. And we can also obtain that

Lemma 2.2 Let $G$ be a simple graph and $H$ be a subgraph(resp. proper subgraph) of $G$. Then $G \succeq H($ resp. $\succ H)$. 
A connected graph with $n$ vertices and $n$ edges is called a unicyclic graph. Obviously, a unicyclic graph has exactly one cycle. A connected graph with $n$ vertices and $n+1$ edges is called a bicyclic graph. Let $\mathcal{U}(n)$ be the class of connected unicyclic graphs with $n$ vertices, $\mathcal{U}(n, d)$ be the class of unicyclic graphs with $n$ vertices and diameter $d$, where $1 \leq d \leq n-2$. Let $\mathcal{B}(n)$ be the class of bicyclic graphs with $n$ vertices and $\mathcal{B}(n, d)$ be the class of bicyclic graphs in $\mathcal{B}(n)$ with diameter $d$, where $2 \leq d \leq n-2$. Let $P_{n}$ be the path with $n$ vertices and $K_{n}$ be the complete graph with $n$ vertices.

When $d=1, n=3, K_{3}$ is the unique graph in $\mathcal{U}(3,1)$. When $d=1$, $n \geq 4, \mathcal{U}(n, 1)$ contains no graphs. When $d=2, n=4, \mathcal{U}(4,2)$ has two graphs $G_{4,2}^{1}$ and $G_{4,2}^{2}$ (see Figure 1). Clearly, $\operatorname{ME}\left(G_{4,2}^{2}\right)>M E\left(G_{4,2}^{1}\right)$, i.e., $G_{4,2}^{1}$ is the unique graph with minimal matching energy in $\mathcal{U}(4,2)$. When $d=2, n \geq 5$, the graph obtained by attaching $n-3$ pendant vertices to a vertex of a triangle is the unique graph in $\mathcal{U}(n, 2)$. Thus, we just consider the case in which $3 \leq d \leq n-2$. In section 3 of our paper, we will prove that for $3 \leq d \leq n-2$, the graph $U_{n, d}$ is the unique graph in $\mathcal{U}(n, d)$ with minimal matching energy, where the graph $U_{n, d}$ is shown in Figure 2.

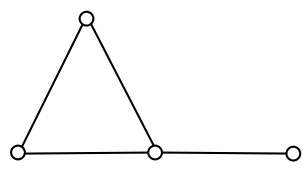

$G_{4,2}^{1}$

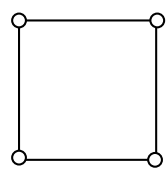

$G_{4,2}^{2}$

Figure 1: The two graphs in $\mathcal{U}(4,2)$.

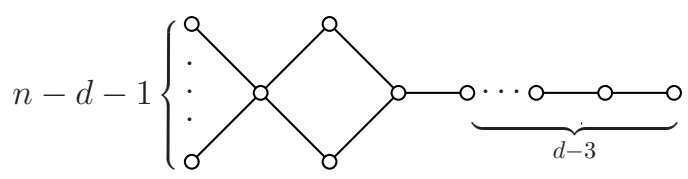

$U_{n, d}$ with $3 \leq d \leq n-2$

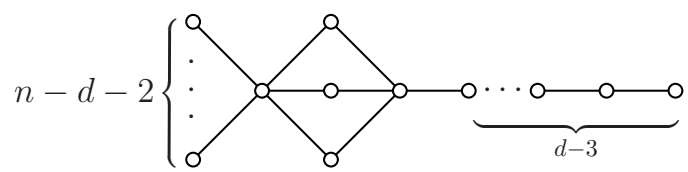

$$
B_{n, d} \text { with } 3 \leq d \leq n-3
$$

Figure 2: The graphs $U_{n, d}$ and $B_{n, d}$. 
When $d=2, n \geq 6, \mathcal{B}(n, 2)$ has two graphs $G_{n, 2}^{1}$ and $G_{n, 2}^{2}$ (see Figure 3). By Lemma 2.1 and simple calculation, we can get $G_{n, 2}^{1} \succ G_{n, 2}^{2}$, hence $G_{n, 2}^{2}$ is the unique graph in $\mathcal{B}(n, 2)$ with minimal matching energy. Therefore, we only consider the case in which $3 \leq d \leq n-2$. In section 4 , we will show that $B_{n, d}$ is the unique graph with minimal matching energy for $3 \leq d \leq n-3$, where the graph $B_{n, d}$ is shown in Figure 2, Furthermore, we also pay our attention to the case $d=n-2$.

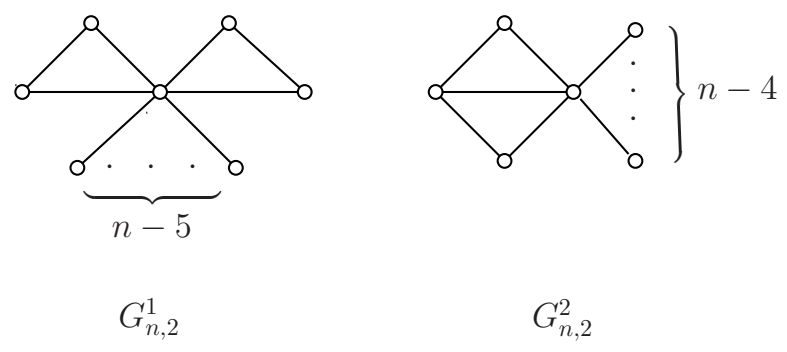

Figure 3: The two graphs in $\mathcal{B}(n, 2)$.

Let $\mathcal{T}(n, d)$ be the class of trees with $n \geq 2$ vertices and diameter $d$, where $1 \leq d \leq n-1$. If $T \in \mathcal{T}(n, 1)$, then $T=P_{2}$. For $1 \leq d \leq n-1$, let $T_{n, d}$ denote the graph obtained by attaching $n-d$ pendent vertices to an end vertex of $P_{d}$. Specially, $T_{n, 1}=T_{n, 2}=S_{n}$. Obviously, $T_{n, 2}$ is the unique tree in $\mathcal{T}(n, 2)$ and $T_{n, n-1}=P_{n}$ is the unique tree in $\mathcal{T}(n, n-1)$.

Let $G_{1}, G_{2}$ be two graphs with $n$ vertices. Now we introduce a quasiorder $\succeq_{1}$ defined in [31]: If $b_{i}\left(G_{1}\right) \geq b_{i}\left(G_{2}\right)$ for all $i \geq 0$, then we write $G_{1} \succeq_{1} G_{2}$. If $G_{1} \succeq_{1} G_{2}$ and there exists an $i_{0}$ such that $b_{i_{0}}\left(G_{1}\right)>b_{i_{0}}\left(G_{2}\right)$, then we write $G_{1} \succ_{1} G_{2}$. The following lemmas are relevant results on this quasi-order.

Lemma $2.3([22,40])$ For $2 \leq i \leq\left\lfloor\frac{n}{2}\right\rfloor$ and $n \geq 4$,

$$
P_{n} \succ_{1} P_{i} \cup P_{n-i} \succ_{1} P_{1} \cup P_{n-1} .
$$

Lemma 2.4 ([19]) For $3 \leq d \leq n-2, P_{n} \succeq_{1} T_{n, d} \succeq_{1} S_{n}$.

Lemma $2.5([37])$ Let $T \in \mathcal{T}(n, d)$ and $T \neq T_{n, d}$. Then $T \succ_{1} T_{n, d}$.

Lemma 2.6 ([29]) If $d>d_{0} \geq 3$, then $T_{n, d} \succ_{1} T_{n, d_{0}}$.

Lemma 2.7 ([39]) For $2 \leq d_{1} \leq n_{1}-2$, we have $T_{n_{1}, d_{1}} \cup T \succeq_{1} T_{n_{1}+n_{2}-1, d_{1}+d_{2}}$, where $T=T_{n_{2}, d_{2}}$ if $2 \leq d_{2} \leq n_{2}-2$, and $P_{2}$ if $n_{2}=2$ and $d_{2}=1$.

If $G$ is an acyclic graph, then [22] $b_{2 k}(G)=m_{k}(G)$ and $b_{2 k+1}(G)=0$ for all $k$. Thus, the quasi-order $\succ_{1}$ (resp. $\succeq_{1}$ ) in Lemmas 2.3 2.7 can be replaced by $\succ$ (resp. $\succeq)$, and the results also work. 
By Lemma 2.1 and the definition of the quasi-order $\succeq$, it is easy to see that the following lemma holds.

Lemma 2.8 Let $G, G^{\prime} \in \mathcal{U}(n)$ and uv(resp. $\left.u^{\prime} v^{\prime}\right)$ be a pendant edge with the pendant vertex $u\left(\right.$ resp. $\left.u^{\prime}\right)$ of the graph $G$ (resp. $\left.G^{\prime}\right)$. If $G-u \succeq G^{\prime}-u^{\prime}$ and $G-u-v \succ G^{\prime}-u^{\prime}-v^{\prime}$, or $G-u \succ G^{\prime}-u^{\prime}$ and $G-u-v \succeq G^{\prime}-u^{\prime}-v^{\prime}$, then $G \succ G^{\prime}$.

The following lemmas will be needed in our paper, which are obtained based on the previous results.

Lemma 2.9 For $3 \leq d \leq n-2, B_{n, d} \succ U_{n, d} \succ T_{n, d}$.

Proof. Since $U_{n, d}$ is a proper subgraph of $B_{n, d}$, then by Lemma 2.2, we can get $B_{n, d} \succ U_{n, d}$. Similarly, we also have $U_{n, d} \succ T_{n, d}$.

Lemma 2.10 For $3 \leq d_{0}<d \leq n-2, U_{n, d} \succ U_{n, d_{0}}$.

Proof. By Lemmas 2.1, 2.2 and 2.6,

$$
\begin{aligned}
m_{k}\left(U_{n, d}\right) & \geq m_{k}\left(U_{n-1, d-1}\right)+m_{k-1}\left(T_{n-2, d-2}\right) \\
& \geq m_{k}\left(U_{n-1, d-1}\right)+m_{k-1}\left(T_{d-1, d-3}\right) \\
& =m_{k}\left(U_{n, d-1}\right) .
\end{aligned}
$$

Furthermore, $m_{2}\left(U_{n, d}\right)>m_{2}\left(U_{n, d-1}\right)$. It follows that $U_{n, d} \succ U_{n, d-1}$. Therefore, $U_{n, d} \succ U_{n, d-1} \succ \cdots \succ U_{n, d_{0}}$.

Similarly, we have

Lemma 2.11 For $3 \leq d_{0}<d \leq n-2, B_{n, d} \succ B_{n, d_{0}}$.

\section{$3 \quad$ Unicyclic graphs with a given diameter}

Now we consider the minimal matching energy of graphs in $\mathcal{U}(n, d)$ with $3 \leq d \leq n-2$. We first discuss the case $d=n-2$.

Lemma 3.1 Let $G \in \mathcal{U}(n, n-2)$ with $n \geq 8$ and $G \neq U_{n, n-2}$. Then $G \succ U_{n, n-2}$.

Proof. We will prove the lemma by induction on $n$.

If $n=8$, then $G$ is isomorphic to one of the following graphs (see Figure (4).

It is easy to get

$$
\begin{aligned}
& \alpha\left(U_{8,6}^{1}\right)=\mu^{8}-8 \mu^{6}+19 \mu^{4}-13 \mu^{2}+1 ; \quad \alpha\left(U_{8,6}^{2}\right)=\mu^{8}-8 \mu^{6}+18 \mu^{4}-11 \mu^{2}+1 ; \\
& \alpha\left(U_{8,6}^{3}\right)=\mu^{8}-8 \mu^{6}+18 \mu^{4}-12 \mu^{2}+1 ; \quad \alpha\left(U_{8,6}^{4}\right)=\mu^{8}-8 \mu^{6}+19 \mu^{4}-14 \mu^{2}+2 ;
\end{aligned}
$$




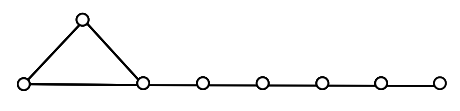

$U_{8,6}^{1}$

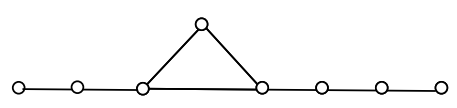

$U_{8,6}^{3}$

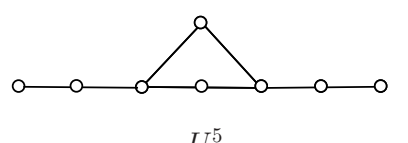

$U_{8,6}^{5}$

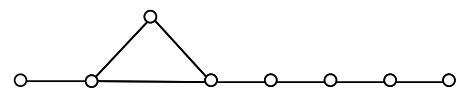

$U_{8,6}^{2}$

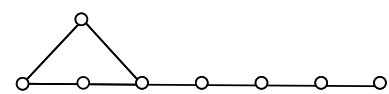

$U_{8,6}^{4}$

Figure 4: The graphs in $\mathcal{U}(8,6)$ except for $U_{8,6}$.

$\alpha\left(U_{8,6}^{5}\right)=\mu^{8}-8 \mu^{6}+18 \mu^{4}-12 \mu^{2}+2 ; \quad \alpha\left(U_{8,6}\right)=\mu^{8}-8 \mu^{6}+18 \mu^{4}-11 \mu^{2}$.

And then it is obvious that $U_{8,6}^{i} \succ U_{8,6}$ for $i=1,2,3,4,5$, i.e., $U_{8,6}$ is the unique graph in $\mathcal{U}(8,6)$ with minimal matching energy. 5).

If $n=9$, then $G$ is isomorphic to one of the following graphs (see Figure
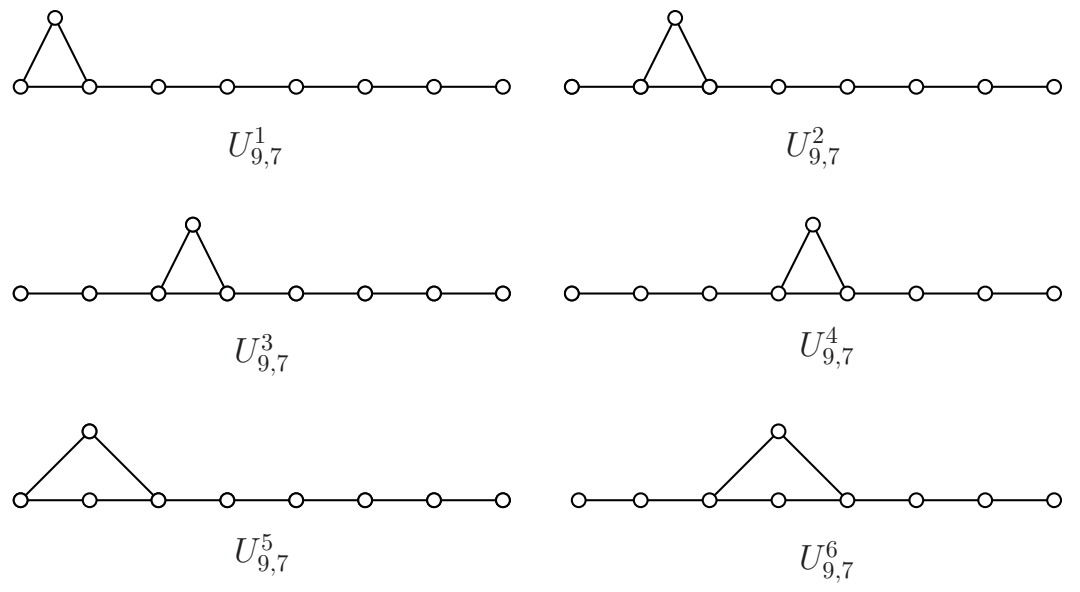

Figure 5: The graphs in $\mathcal{U}(9,7)$ except for $U_{9,7}$.

We can obtain that

$$
\begin{array}{ll}
\alpha\left(U_{9,7}^{1}\right)=\mu^{9}-9 \mu^{7}+26 \mu^{5}-26 \mu^{3}+6 \mu ; & \alpha\left(U_{9,7}^{2}\right)=\mu^{9}-9 \mu^{7}+25 \mu^{5}-23 \mu^{3}+5 \mu ; \\
\alpha\left(U_{9,7}^{3}\right)=\mu^{9}-9 \mu^{7}+25 \mu^{5}-24 \mu^{3}+6 \mu ; & \alpha\left(U_{9,7}^{4}\right)=\mu^{9}-9 \mu^{7}+25 \mu^{5}-24 \mu^{3}+5 \mu ; \\
\alpha\left(U_{9,7}^{5}\right)=\mu^{9}-9 \mu^{7}+26 \mu^{5}-27 \mu^{3}+8 \mu ; & \alpha\left(U_{9,7}^{6}\right)=\mu^{9}-9 \mu^{7}+25 \mu^{5}-24 \mu^{3}+6 \mu ;
\end{array}
$$


along with

$$
\alpha\left(U_{9,7}\right)=\mu^{9}-9 \mu^{7}+25 \mu^{5}-23 \mu^{3}+4 \mu .
$$

It now immediately follows that $U_{9,7}^{i} \succ U_{9,7}$ for $i=1,2,3,4,5,6$, i.e., $U_{9,7}$ is the unique graph in $\mathcal{U}(9,7)$ with minimal matching energy.

Now suppose that the result holds for graphs in $\mathcal{U}(n-1, n-3)$ and $\mathcal{U}(n-2, n-4)$. Let $G \in \mathcal{U}(n, n-2)$ and $G \neq U_{n, n-2}$, where $n \geq 10$.

Let $u$ (resp. $u^{\prime}$ ) be a pendant vertex, adjacent to $v$ (resp. $\left.v^{\prime}\right)$, which has the largest distance to a vertex on the unique cycle of $G$ (resp. $\left.U_{n, n-2}\right)$. Then the degree of $v$ is 2. So is $v^{\prime}$. Hence $G-u \in \mathcal{U}(n-1, n-3), G-u-v \in$ $\mathcal{U}(n-2, n-4)$ and $U_{n, n-2}-u^{\prime}=U_{n-1, n-3}, U_{n, n-2}-u^{\prime}-v^{\prime}=U_{n-2, n-4}$.

Since $G \neq U_{n, n-2}$, we have either $G-u \neq U_{n-1, n-3}$ or $G-u-v \neq$ $U_{n-2, n-4}$. By the induction hypothesis, we have $G-u \succ U_{n-1, n-3}$ and $G-u-v \succeq U_{n-2, n-4}$, or $G-u \succeq U_{n-1, n-3}$ and $G-u-v \succ U_{n-2, n-4}$. By Lemma 2.8, $G \succ U_{n, n-2}$.

Theorem 3.2 Let $G \in \mathcal{U}(n, d)$ with $n \geq 8,3 \leq d \leq n-2$ and $G \neq U_{n, d}$. Then $M E(G)>M E\left(U_{n, d}\right)$.

Proof. We prove the result by induction on $n-d$.

When $n-d=2$, by Lemma 3.1, we have $G \succ U_{n, d}$. Let $t \geq 3$ and suppose that the result holds for $n-d<t$. Now suppose that $n-d=t$. Let $u^{\prime}$ be the vertex of degree 3 in $U_{n, d}$ and $v^{\prime}$ be a vertex on the quadrangle that is adjacent to $u^{\prime}$. By Lemma 2.1.

$$
\begin{aligned}
m_{k}\left(U_{n, d}\right) & =m_{k}\left(U_{n, d}-u^{\prime} v^{\prime}\right)+m_{k-1}\left(U_{n, d}-u^{\prime}-v^{\prime}\right) \\
& =m_{k}\left(T_{n, d}\right)+m_{k-1}\left(P_{d-3} \cup S_{n-d+1}\right) .
\end{aligned}
$$

For $C_{n}, m_{k}\left(C_{n}\right)=m_{k}\left(P_{n}\right)+m_{k-1}\left(P_{n-2}\right)$. By Lemma 2.4. $P_{n} \succ T_{n, d}$. And by Lemmas 2.3 and 2.4. $P_{n-2} \succeq P_{d-3} \cup P_{n-d+1} \succ P_{d-3} \cup S_{n-d+1}$. Thus $C_{n} \succ U_{n, d}$. Therefore, we may suppose that the unique cycle of $G$ is $C_{r}$ with $r<n$. Let $P(G)=v_{0} v_{1} \ldots v_{d}$ be a diametrical path of $G$. Then one of $v_{0}$ and $v_{d}$ must be a pendant vertex.

Case 1 All pendant vertices are on $P(G)$.

Since $t=n-d \geq 3$, then $|V(P(G))|=d+1 \leq n-2$. Thus there are at least two adjacency vertices, say $u$ and $v$, on $C_{r}$ which lie outside $P(G)$ such that $G-u v \in \mathcal{T}\left(n, d_{1}\right)$, and $G-u-v \in \mathcal{T}\left(n-2, d_{2}\right)$, where $d_{1}, d_{2} \geq d$. By Lemmas 2.5 and 2.6. $G-u v \succeq T_{n, d_{1}} \succeq T_{n, d}, G-u-v \succeq T_{n-2, d_{2}} \succeq T_{n-2, d}$. We also have $T_{n-2, d} \succ P_{d-3} \cup T_{n-d+1,3} \succ P_{d-3} \cup S_{n-d+1}$ by Lemmas 2.2 and 2.4. Hence, $G-u-v \succ P_{d-3} \cup S_{n-d+1}$.

Note that $m_{k}(G)=m_{k}(G-u v)+m_{k-1}(G-u-v)$, so $m_{k}(G) \geq m_{k}\left(U_{n, d}\right)$ for all $k$. Moreover, since $T_{n-2, d} \succ P_{d-3} \cup S_{n-d+1}$, there exists some $k_{0}$ such that $m_{k_{0}-1}(G-u-v)>m_{k_{0}-1}\left(P_{d-3} \cup S_{n-d+1}\right)$, i.e., $m_{k_{0}}(G)>m_{k_{0}}\left(U_{n, d}\right)$. Thus $G \succ U_{n, d}$.

Case 2 There is at least one pendant vertex outside $P(G)$. 
Let $u^{\prime}$ be a pendant vertex of $U_{n, d}$ adjacent to the vertex $v^{\prime}$ of degree $n-d+1$. Then $U_{n, d}-u^{\prime}=U_{n-1, d}$, and $U_{n, d}-u^{\prime}-v^{\prime}=(n-d-2) P_{1} \cup T_{d, d-2}$.

Subcase 2.1 There is a pendant vertex $u$ outside $P(G)$ such that its neighbor $v$ lies on $C_{r}$.

Since $u$ outside $P(G)$, then $G-u \in \mathcal{U}(n-1, d)$. Consequently, by the induction hypothesis, $G-u \succeq U_{n-1, d}$.

If $v$ lies outside $P(G)$, then $G-u-v \supseteq P_{d+1}$. Thus $G-u-v \succeq P_{d+1} \succ$ $T_{d, d-2}$.

Suppose that $v$ lies on $P(G)$, then $P(G)$ and $C_{r}$ have common vertices, say $v_{i}, \ldots, v_{i+j}$ with $j \geq 0$.

If $j=0$, i.e., $v=v_{i}$ is the unique common vertex of $P(G)$ and $C_{r}$, then $G-u-v \supseteq P_{i} \cup P_{d-i} \cup P_{2}$. Since

$$
m_{k}\left(P_{i} \cup P_{d-i} \cup P_{2}\right) \geq m_{k}\left(P_{d}\right) \geq m_{k}\left(T_{d, d-2}\right)
$$

and $m_{2}\left(P_{i} \cup P_{d-i} \cup P_{2}\right)>m_{2}\left(T_{d, d-2}\right)$, then $P_{i} \cup P_{d-i} \cup P_{2} \succ T_{d, d-2}$. Therefore, $G-u-v \succeq P_{i} \cup P_{d-i} \cup P_{2} \succ T_{d, d-2}$.

If $j>0$. For $v \neq v_{i}, v_{i+j}, G-u-v \supseteq P_{d+1}$. So $G-u-v \succeq P_{d+1} \succ T_{d, d-2}$. Otherwise, for $v=v_{i}$ or $v_{i+j}$, say $v=v_{i}$. Then $G-u-v \supseteq P_{i} \cup T_{1}$, where $T_{1} \in \mathcal{T}(d-i+1, d-i-1)$ is obtained by attaching a pendant vertex to vertex $v_{i+j}$ of the path $P=v_{i+1} \cdots v_{d}$. For $k \geq 0$,

$$
m_{k}\left(P_{i} \cup T_{1}\right) \geq m_{k}\left(P_{i} \cup T_{d-i+1, d-i-1}\right) \geq m_{k}\left(T_{d, d-2}\right) .
$$

If $(i, j) \neq(1,2)$, then $m_{2}\left(P_{i} \cup T_{1}\right)>m_{2}\left(T_{d, d-2}\right)$, hence $G-u-v \succeq P_{i} \cup$ $T_{1} \succ T_{d, d-2}$. Otherwise, $P_{i} \cup T_{1}$ is a proper subgraph of $G-u-v$, then $G-u-v \succ P_{i} \cup T_{1} \succeq T_{d, d-2}$. Thus we always have $G-u-v \succ T_{d, d-2}$. Therefore, $G-u-v \succ U_{n, d}-u^{\prime}-v^{\prime}$.

We have proved that $G-u \succeq U_{n-1, d}$. Then by Lemma 2.8, we obtain $G \succ U_{n, d}$.

Subcase 2.2 The neighbor of any pendant vertex outside $P(G)$ also lies outside $C_{r}$.

If there is a pendant vertex $u$ such that its neighbor $v$ lies outside $P(G)$, then $G-u-v \supseteq C_{r} \cup P_{d+1} \supseteq C_{r} \cup P_{j} \cup P_{d-j}$ or $G-u-v \supseteq G^{\prime}$, where $G^{\prime} \in \mathcal{U}(s, d)$ with $d+2 \leq s \leq n-2$.

If every pendant vertex outside $P(G)$ is adjacent to a vertex on $P(G)$, then we choose a pendant vertex $u$, adjacent to $v=v_{j}$ such that $G-u-v \supseteq$ $C_{r} \cup P_{j} \cup P_{d-j}$ or $G-u-v \supseteq P_{j} \cup G^{\prime \prime}$, where $G^{\prime \prime} \in \mathcal{U}\left(s^{\prime}, d^{\prime}\right)$ with $d^{\prime} \geq d-j-1$, $s^{\prime} \geq d^{\prime}+2$ and $s^{\prime}+j \leq n-2$.

Hence there are three possibilities: $G-u-v \supseteq C_{r} \cup P_{j} \cup P_{d-j}, G-u-v \supseteq$ $G^{\prime}$ or $G-u-v \supseteq P_{j} \cup G^{\prime \prime}$.

First, suppose that $G-u-v \supseteq C_{r} \cup P_{j} \cup P_{d-j}$, then

$$
m_{k}\left(C_{r} \cup P_{j} \cup P_{d-j}\right) \geq m_{k}\left(P_{3} \cup P_{j} \cup P_{d-j}\right) \geq m_{k}\left(T_{d, d-2}\right) .
$$


In particular, $m_{1}\left(C_{r} \cup P_{j} \cup P_{d-j}\right)>m_{1}\left(T_{d, d-2}\right)$. Thus, $G-u-v \succeq C_{r} \cup$ $P_{j} \cup P_{d-j} \succ T_{d, d-2}$.

Next, suppose that $G-u-v \supseteq G^{\prime}$, then $G-u-v \succeq U_{s, d} \succeq U_{d+2, d} \succ$ $T_{d+2, d}$.

Finally, suppose that $G-u-v \supseteq P_{j} \cup G^{\prime \prime}$. For $G^{\prime \prime} \in \mathcal{U}\left(s^{\prime}, d^{\prime}\right)$ with $s^{\prime}-d^{\prime} \leq n-2-j-(d-j-1)=n-d-1$. By the induction hypothesis, $G^{\prime \prime} \succeq U_{s^{\prime}, d^{\prime}} \succeq U_{d-j+1, d-j-1}$. Thus

$$
G-u-v \succeq P_{j} \cup G^{\prime \prime} \succeq P_{j} \cup U_{s^{\prime}, d^{\prime}} \succeq P_{j} \cup U_{d-j+1, d-j-1} .
$$

For $k \geq 0$,

$$
m_{k}\left(P_{j} \cup U_{d-j+1, d-j-1}\right) \geq m_{k}\left(P_{j} \cup T_{d-j+1, d-j-1}\right) \geq m_{k}\left(T_{d, d-2}\right) .
$$

Furthermore, $m_{1}\left(P_{j} \cup U_{d-j+1, d-j-1}\right)>m_{1}\left(T_{d+2, d}\right)$. It follows that $G-u-v \succeq$ $P_{j} \cup U_{d-j+1, d-j-1} \succ T_{d, d-2}$.

According to the arguments above, we have proved that $G-u-v \succ$ $U_{n, d}-u^{\prime}-v^{\prime}$. On the other hand, $G-u \succeq U_{n-1, d}$. Thus by Lemma 2.8, $G \succ U_{n, d}$.

Combining Cases 1 and 2, we conclude that $G \succ U_{n, d}$ also holds for $G \in \mathcal{U}(n, d)$ with $3 \leq d \leq n-3$ and $G \neq U_{n, d}$, which yields the result.

\section{Bicyclic graphs with a given diameter}

In what follows we state some new definitions and notations. For a graph $G \in \mathcal{B}(n)$, it has either two or three distinct cycles. If $G$ has exactly two cycles, suppose that the lengths of them are $a$ and $b$ respectively. If $G$ has three cycles, then any two cycles must have at least one edge in common, and we may choose two cycles of lengths of $a$ and $b$ with $t$ common edges such that $a-t \geq t$ and $b-t \geq t$. Then, in any case, we choose two cycles $C_{a}$ and $C_{b}$ in $G$. For convenience, let $C_{a}=v_{0} v_{1} \cdots v_{a-1} v_{0}$ and $C_{b}=$ $u_{0} u_{1} \cdots u_{b-1} u_{0}$. If $C_{a}$ and $C_{b}$ have no common edges, then $C_{a}$ and $C_{b}$ are connected by a unique path $P$, say from $v_{0}$ to $u_{0}$. Let $l(G)$ be the length of $P$. If $C_{a}$ and $C_{b}$ have exactly $t(\geq 1)$ common edges, and thus have exactly $t+1$ common vertices, say, $v_{0}=u_{0}, v_{1}=u_{1}, \ldots, v_{t}=u_{t}$, then $C_{c}=u_{0} u_{b-1} \cdots u_{t+1} u_{t} v_{t+1} v_{t+2} \cdots v_{a-1} v_{0}$ is the third cycle of $G$, where $c=b+a-2 t$. If we write $w_{0}=u_{0}, w_{1}=u_{b-1}, \ldots, w_{c-1}=v_{a-1}$, then $C_{c}=w_{0} w_{1} \cdots w_{c-1} w_{0}$. Denote by $d(G)$ the diameter of $G$.

Now we turn our attention to the minimal matching energy of graphs in $\mathcal{B}(n, d)$ with $3 \leq d \leq n-2$. We first deal with the case $d=n-3$.

Lemma 4.1 Let $G \in \mathcal{B}(n, n-3)$ with $n \geq 7$, and $G \neq B_{n, n-3}$. Then $G \succ B_{n, n-3}$. 
Proof. By induction on $n$ to prove this fact.

For $n=7$ and $n=8$, there are only finitely many graphs we need to consider. Then by Lemma 2.1 and direct check, we can get $G \succ B_{n, n-3}$.

Suppose that the result holds for all graphs in $\mathcal{B}(n-1, n-4)$ and $\mathcal{B}(n-$ $2, n-5)$, where $n \geq 9$. Let $G \in \mathcal{B}(n, n-3)$ and $G \neq B_{n, n-3}$.

Case 1 There is a pendent vertex $u$ in $G$ such that the degree of its neighbor $v$ is 2 .

In this case, $G-u \in \mathcal{B}(n-1, n-4)$ and $G-u-v \in \mathcal{B}(n-2, n-5)$. Since $G \neq B_{n, n-3}$, then $G-u \neq B_{n-1, n-4}$ or $G-u-v \neq B_{n-2, n-5}$. By the induction hypothesis, $G-u \succ B_{n-1, n-4}$ and $G-u-v \succeq B_{n-2, n-5}$, or $G-u \succeq B_{n-1, n-4}$ and $G-u-v \succ B_{n-2, n-5}$. Hence, $G \succ B_{n, n-3}$.

Case 2 The neighbor of any pendent vertex has degree at least 3 or there is no pendent vertex.

Then $G$ is isomorphic to some $H_{j}, j=1,2$ (see Figure 6), or $G$ contains one triangle or one quadrangle which has at most one common vertex with the other cycle that is a triangle or a quadrangle.

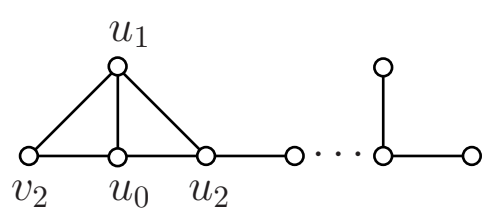

$H_{1}$

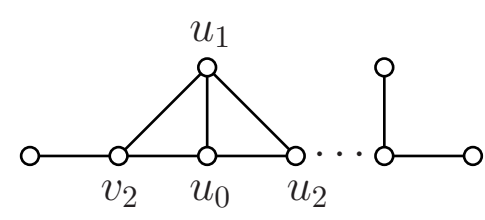

$\mathrm{H}_{2}$

Figure 6: The graphs $H_{i}$ for $i=1,2$.

If $G$ is isomorphic to $H_{1}$, then by Lemmas 2.1, 2.5, 2.6 and Theorem 3.2,

$$
\begin{aligned}
m_{k}(G) & =m_{k}\left(G-u_{1} v_{2}\right)+m_{k-1}\left(G-u_{1}-v_{2}\right) \\
& \geq m_{k}\left(U_{n, n-3}\right)+m_{k-1}\left(T_{n-2, d-1}\right) \\
& \geq m_{k}\left(U_{n, n-3}\right)+m_{k-1}\left(P_{n-6} \cup S_{4}\right) \\
& =m_{k}\left(B_{n, n-3}\right) .
\end{aligned}
$$

Moreover, $m_{2}(G)>m_{2}\left(B_{n, n-3}\right)$, thus $G \succ B_{n, n-3}$.

If $G$ is isomorphic to $H_{2}$, then by Lemmas 2.1, 2.3 and Theorem 3.2 ,

$$
\begin{aligned}
m_{k}(G) & =m_{k}\left(G-u_{1} u_{0}\right)+m_{k-1}\left(G-u_{1}-u_{0}\right) \\
& \geq m_{k}\left(U_{n, n-3}\right)+m_{k-1}\left(P_{2} \cup T_{n-4, n-6}\right) \\
& \geq m_{k}\left(U_{n, n-3}\right)+m_{k-1}\left(P_{2} \cup P_{n-5}\right)+m_{k-2}\left(P_{2} \cup P_{n-7}\right) \\
& \geq m_{k}\left(U_{n, n-3}\right)+m_{k-1}\left(P_{3} \cup P_{n-6}\right)+m_{k-2}\left(P_{n-6}\right) \\
& =m_{k}\left(B_{n, n-3}\right) .
\end{aligned}
$$

Similarly, $m_{2}(G)>m_{2}\left(B_{n, n-3}\right)$, thus $G \succ B_{n, n-3}$. 


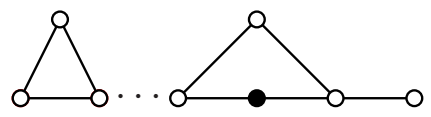

$G_{1}$

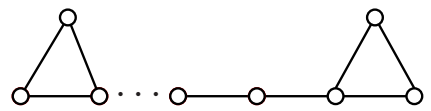

$G_{3}$

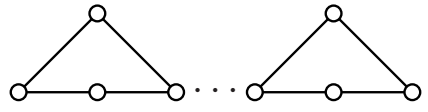

$G_{5}$

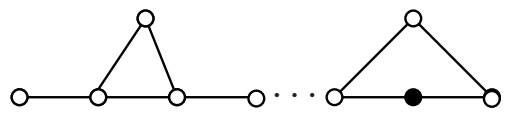

$G_{2}$

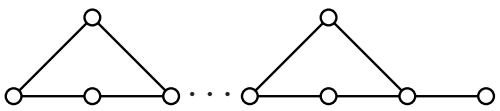

$G_{4}$

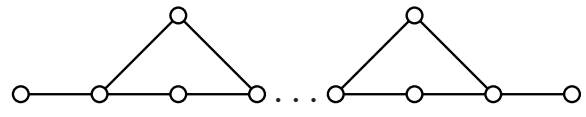

$G_{6}$

Figure 7: The graphs $G_{i}$ for $i=1,2,3,4,5,6$.

Otherwise, $G$ contains one triangle or one quadrangle which has at most one common vertex with the other cycle that is a triangle or a quadrangle. Choose $C_{a}$ and $C_{b}$ as above. Let $b \geq a$.

If $a=3$, then $G$ is isomorphic to $G_{1}, G_{2}$ or $G_{3}$ in Figure 7 , where the black vertices may not occur. Similarly, we can obtain that $G \succ B_{n, n-3}$.

If $a=4$, then $G$ is isomorphic to $G_{4}, G_{5}$ or $G_{6}$ in Figure 7 We can show that $G \succ B_{n, n-3}$ in the same way.

Hence the conclusion follows.

Lemma 4.2 Let $G \in \mathcal{B}(n, d)$ with $n \geq 8$ and $3 \leq d \leq n-4$. If $G$ contains no pendent vertices, then $G \succ B_{n, d+1}$.

Proof. We choose $C_{a}, C_{b}$ in $G$ and if there exists the third cycle, then we choose $C_{c}$ and $t$ as above. Let $b \geq a$. Since $d \leq n-4$, we have $b \geq 5$.

Case $1 C_{a}$ and $C_{b}$ have no common edges.

Then $d=\left\lfloor\frac{a}{2}\right\rfloor+\left\lfloor\frac{b}{2}\right\rfloor+l(G), d\left(G-u_{1} u_{2}\right)=\left\lfloor\frac{a}{2}\right\rfloor+l(G)+b-2 \geq d+1$, $d\left(G-u_{1}-u_{2}\right)=\left\lfloor\frac{a}{2}\right\rfloor+l(G)+b-3 \geq d$. According to Lemmas 2.1, 2.2, 
2.10 and Theorem 3.2,

$$
\begin{aligned}
m_{k}(G) & =m_{k}\left(G-u_{1} u_{2}\right)+m_{k-1}\left(G-u_{1}-u_{2}\right) \\
& \geq m_{k}\left(U_{n, d+1}\right)+m_{k-1}\left(U_{n-2, d}\right) \\
& \geq m_{k}\left(U_{n, d+1}\right)+m_{k-1}\left(P_{d-2} \cup S_{n-d}\right) \\
& =m_{k}\left(B_{n, d+1}\right) .
\end{aligned}
$$

Further, we have $m_{2}(G)>m_{2}\left(B_{n, d+1}\right)$, thus $G \succ B_{n, d+1}$.

Case $2 C_{a}$ and $C_{b}$ have at least one common edge.

Notice that $a-t \geq t, b-t \geq t$, where $t \geq 1$. It follows that $c \geq b+1$, $d=\left\lfloor\frac{c}{2}\right\rfloor=\left\lfloor\frac{(a+b)}{2}\right\rfloor-t, d\left(G-w_{0}-w_{1}\right)=c-3 \geq d$ and $d \geq 3$.

If $b>5$ or $b=5$ and $a$ is even, then $d\left(G-w_{0} w_{1}\right)=\left\lfloor\frac{a}{2}\right\rfloor+b-t-1 \geq d+1$, by Lemmas 2.1, 2.2, 2.9 and Theorem 3.2 .

$$
\begin{aligned}
m_{k}(G) & =m_{k}\left(G-w_{0} w_{1}\right)+m_{k-1}\left(G-w_{0}-w_{1}\right) \\
& \geq m_{k}\left(U_{n, d+1}\right)+m_{k-1}\left(U_{n-2, d}\right) \\
& \geq m_{k}\left(U_{n, d+1}\right)+m_{k-1}\left(T_{n-2, d}\right) \\
& \geq m_{k}\left(U_{n, d+1}\right)+m_{k-1}\left(P_{d-2} \cup S_{n-d}\right) \\
& =m_{k}\left(B_{n, d+1}\right)
\end{aligned}
$$

together with $m_{2}(G)>m_{2}\left(B_{n, d+1}\right)$, hence $G \succ B_{n, d+1}$.

If $b=5$ and $a$ is odd, then $G$ is isomorphic to the two graphs in Figure 8 , it is easy to verify that $G \succ B_{n, d+1}$ and the proof is complete.
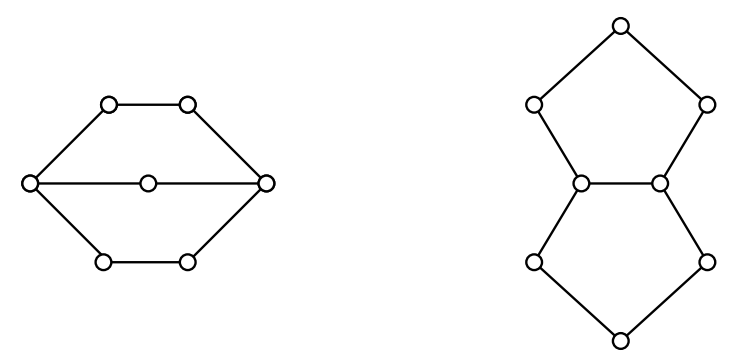

Figure 8: The graphs isomorphic to $G$ when $b=5$ and $a$ is odd.

Lemma 4.3 Let $G \in \mathcal{B}(n, d)$ with $n \geq 8$ and $3 \leq d \leq n-4$. If $G$ contains exactly one pendent vertex $u$ on all diametrical paths of $G$ such that $G-u$ contains no pendent vertices, then $G \succ B_{n, d+1}$.

Proof. We choose $C_{a}, C_{b}$ in $G$ and if there exists the third cycle, then we choose $C_{c}$ and $t$ as above. Let $b \geq a$. Since $d \leq n-4$, we have $b \geq 5$. Let $v$ be the neighbor of $u$.

Case $1 C_{a}$ and $C_{b}$ have no common edges. 
Then $d=\left\lfloor\frac{a}{2}\right\rfloor+\left\lfloor\frac{b}{2}\right\rfloor+l(G)+1$.

If $b \geq 7$, then $d\left(G-u_{1} u_{2}\right) \geq\left\lfloor\frac{a}{2}\right\rfloor+l(G)+b-2 \geq d+1$.

If $v$ lies on $C_{a}$ and $b=5$ or 6 , then $d\left(G-u_{1} u_{2}\right)=\left\lfloor\frac{a}{2}\right\rfloor+l(G)+b-1=d+1$.

If $v$ lies on $C_{b}, a=5$ and $b=6$, then $d\left(G-v_{1} v_{2}\right)=\left\lfloor\frac{b}{2}\right\rfloor+l(G)+a-1=$ $d+1$.

In these cases, the proof is the same as Case 1 of Lemma 4.2 .

Otherwise, $v$ lies on $C_{b}, a=3$ or 4 and $b=5$ or 6 .

If $l(G)=0$, then $G$ is isomorphic to finitely many graphs. Apply Lemma 2.1 and direct calculation, we can get $G \succ B_{n, d+1}$.

So suppose that $l(G) \geq 1$. If $a=3$ and $b=5$, then $G-v_{0} v_{1} \in \mathcal{U}(n, d+1)$. $G-v_{0}-v_{1}=P_{1} \cup G_{0}$, where $G_{0} \in \mathcal{U}(n-3, d-2)$. Since $d\left(G_{0}\right)=d-2$, $n\left(G_{0}\right)=n-3$, then $n-3=d-2+3$, that is, $n-d=4$, meanwhile, $l(G)-1=d-2-3=d-5$, i.e., $l(G)=d-4$. Thus $G_{0}-u_{0} u_{1} \supseteq$ $P_{d-2} \cup S_{3}, G_{0}-u_{0}-u_{1}=P_{d-5} \cup P_{4}$. Therefore, by Lemmas 2.1, 2.2, 2.3 and Theorem 3.2 ,

$$
\begin{aligned}
m_{k}(G) & =m_{k}\left(G-v_{0} v_{1}\right)+m_{k-1}\left(G-v_{0}-v_{1}\right) \\
& \geq m_{k}\left(U_{n, d+1}\right)+m_{k-1}\left(G_{0}-u_{0} u_{1}\right)+m_{k-2}\left(G_{0}-u_{0}-u_{1}\right) \\
& \geq m_{k}\left(U_{n, d+1}\right)+m_{k-1}\left(P_{d-2} \cup S_{3}\right)+m_{k-2}\left(P_{d-2}\right) \\
& \geq m_{k}\left(U_{n, d+1}\right)+m_{k-1}\left(P_{d-2} \cup S_{n-d}\right) \\
& =m_{k}\left(B_{n, d+1}\right) .
\end{aligned}
$$

In particular, $m_{2}(G)>m_{2}\left(B_{n, d+1}\right)$, thus $G \succ B_{n, d+1}$.

If $a=3$ and $b=6$, by similar arguments, we can obtain that $G \succ B_{n, d+1}$.

If $a=4$ and $b=5$, then $n=d+1+3=d+4$, i.e., $n-d-1=3$, $G-v_{0} v_{1} \in \mathcal{U}(n, d+1)$ and $G-v_{0}-v_{1}=P_{2} \cup G_{0}$, where $G_{0} \in \mathcal{U}(n-4, d-3)$. Similarly,

$$
\begin{aligned}
m_{k}(G) & =m_{k}\left(G-v_{0} v_{1}\right)+m_{k-1}\left(G-v_{0}-v_{1}\right) \\
& \geq m_{k}\left(U_{n, d+1}\right)+m_{k-1}\left(P_{2} \cup G_{0}-u_{0} u_{1}\right)+m_{k-2}\left(P_{2} \cup G_{0}-u_{0}-u_{1}\right) \\
& \geq m_{k}\left(U_{n, d+1}\right)+m_{k-1}\left(P_{2} \cup P_{d-2}\right)+m_{k-2}\left(P_{2} \cup P_{d-6} \cup P_{4}\right) \\
& \geq m_{k}\left(U_{n, d+1}\right)+m_{k-1}\left(P_{3} \cup P_{d-3}\right)+m_{k-2}\left(P_{d-2}\right) \\
& =m_{k}\left(B_{n, d+1}\right) .
\end{aligned}
$$

Moreover, $m_{2}(G)>m_{2}\left(B_{n, d+1}\right)$, hence $G \succ B_{n, d+1}$.

If $a=4$ and $b=6$, we can verify that $G \succ B_{n, d+1}$ in the same way.

Case $2 C_{a}$ and $C_{b}$ have at least one common edge.

Then $d=\left\lfloor\frac{c}{2}\right\rfloor+1=\left\lfloor\frac{(a+b)}{2}\right\rfloor-t+1$. Since $b \geq 5, w_{0}, w_{1} \neq v$ and $d\left(G-w_{0} w_{1}\right)=\left\lfloor\frac{a}{2}\right\rfloor+b-t-1$. When $b \geq 6, d\left(G-w_{0} w_{1}\right) \geq d+1$, 
$d\left(G-w_{0} w_{1}\right) \geq d-1$. And then

$$
\begin{aligned}
m_{k}(G) & =m_{k}\left(G-w_{0} w_{1}\right)+m_{k-1}\left(G-w_{0}-w_{1}\right) \\
& \geq m_{k}\left(U_{n, d+1}\right)+m_{k-1}\left(T_{n-2, d-1}\right) \\
& \geq m_{k}\left(U_{n, d+1}\right)+m_{k-1}\left(P_{d-2} \cup S_{n-d}\right) \\
& =m_{k}\left(B_{n, d+1}\right)
\end{aligned}
$$

along with $m_{2}(G)>m_{2}\left(B_{n, d+1}\right)$, hence $G \succ B_{n, d+1}$. Now, we are left with the cases:

(i) $b=5, t=2, a=4, u v \in C_{b}$;

(ii) $b=5, t=2, a=4, u v \in C_{a}$;

(iii) $b=5, t=2, a=5$.

It can be checked directly that $G \succ B_{n, d+1}$ in these cases.

Combining Cases 1 and 2, we arrive at the result.

Theorem 4.4 Let $G \in \mathcal{B}(n, d)$ with $n \geq 8$ and $3 \leq d \leq n-3$. If there are two vertex-disjoint cycles in $G$, then $G \succ B_{n, d}$.

Proof. The proof proceeds by induction on $n-d$. By Lemma 4.1, the result holds for $n-d=3$. Let $h \geq 4$ and assume that the result holds for $n-d<h$. Suppose that $G \in \mathcal{B}(n, d)$ with $n-d=h$.

Case 1 There is no pendent vertex in $G$.

Then by Lemmas 2.11 and 4.2, $G \succ B_{n, d+1} \succ B_{n, d}$.

Case 2 There is a pendent vertex outside some diametrical path $P(G)=$ $x_{0} x_{1} \cdots x_{d}$.

Let $u$, adjacent to $v$, be a pendent vertex outside $P(G)$ in $G$. Then $G-u \in \mathcal{B}(n-1, d)$. Since $(n-1)-d<h$, by the induction hypothesis, $G-u \succ B_{n-1, d}$.

By Lemma 2.1,

$$
m_{k}\left(B_{n, d}\right)=m_{k}\left(B_{n-1, d}\right)+m_{k-1}\left(T_{d+1, d-2}\right)
$$

Meanwhile, let $H=G-u-v$, then

$$
\begin{aligned}
m_{k}(G) & =m_{k}(G-u)+m_{k-1}(G-u-v) \\
& =m_{k}(G-u)+m_{k-1}(H) \\
& \geq m_{k}\left(B_{n-1, d}\right)+m_{k-1}(H) .
\end{aligned}
$$

Hence, to complete the proof we shall show that $m_{k}(H) \geq m_{k}\left(T_{d+1, d-2}\right)$. Select $C_{a}$ and $C_{b}$ as above.

Subcase 2.1 $v$ lies on some cycle, say $C_{a}$. Then $H \supseteq C_{b}$.

First, suppose that $P(G)$ and $C_{b}$ have no common vertices. Then $H \supseteq$ $P_{i} \cup P_{d-i} \cup C_{b}$ when $v$ lies on $P(G)$, say $v=v_{i}$. Otherwise, $H \supseteq P_{d+1} \cup C_{b} \supseteq$ $P_{i} \cup P_{d-i} \cup C_{b}$. Thus

$$
\begin{aligned}
m_{k}(H) & \geq m_{k}\left(P_{i} \cup P_{d-i} \cup C_{b}\right) \geq m_{k}\left(P_{d-1} \cup S_{b}\right) \geq m_{k}\left(P_{d-1} \cup P_{3}\right) \\
& \geq m_{k}\left(P_{d+1}\right) \geq m_{k}\left(T_{d+1, d-2}\right) .
\end{aligned}
$$


Next, suppose that $P(G)$ and $C_{b}$ have common vertices $x_{l}, \ldots, x_{l+q}$, where $q \geq 0$.

If $v$ lies outside $P(G)$, then $H \supseteq G_{1}$, where $G_{1} \in \mathcal{U}\left(s_{1}, d\right)$ with $s_{1} \geq d+2$. Hence,

$$
m_{k}(H) \geq m_{k}\left(G_{1}\right) \geq m_{k}\left(U_{s_{1}, d}\right) \geq m_{k}\left(T_{s_{1}, d}\right) \geq m_{k}\left(P_{d+1}\right) \geq m_{k}\left(T_{d+1, d-2}\right) .
$$

So suppose that $v$ lies on $P(G)$. Then $P(G)$ and $C_{a}$ have common vertices, say $x_{i}, \ldots, x_{i+p}$, where $p \geq 0$.

When $p=0$, then $i \geq 1$, thus $H \supseteq P_{2} \cup P_{i} \cup G_{2}$, where $G_{2} \in \mathcal{U}\left(s_{2}, d_{2}\right)$, $d_{2} \geq d-i-1 \geq 1$ and $s_{2} \geq d_{2}+2$. If $d_{2}=1, i=d-2$, then $G_{2}=C_{3}$. Therefore,

$m_{k}(H) \geq m_{k}\left(P_{2} \cup P_{d-2} \cup C_{3}\right) \geq m_{k}\left(P_{d-1} \cup P_{3}\right) \geq m_{k}\left(P_{d+1}\right) \geq m_{k}\left(T_{d+1, d-2}\right)$.

If $d_{2}=2$, then $s_{2} \geq 4, i \geq d-3$. Consequently,

$$
\begin{aligned}
m_{k}(H) & \geq m_{k}\left(P_{2} \cup P_{i} \cup G_{2}\right) \geq m_{k}\left(P_{2} \cup P_{i} \cup S_{s_{2}}\right) \geq m_{k}\left(P_{2} \cup P_{i} \cup T_{4,2}\right) \\
& \geq m_{k}\left(T_{i+4, i+2}\right) \geq m_{k}\left(T_{d+1, d-1}\right) \geq m_{k}\left(T_{d+1, d-2}\right) .
\end{aligned}
$$

If $d_{2} \geq 3$, then $s_{2} \geq 5$. Thus

$$
\begin{aligned}
m_{k}(H) & \geq m_{k}\left(P_{2} \cup P_{i} \cup G_{2}\right) \geq m_{k}\left(P_{2} \cup P_{i} \cup U_{s_{2}, d_{2}}\right) \geq m_{k}\left(P_{2} \cup P_{i} \cup T_{s_{2}, d_{2}}\right) \\
& \geq m_{k}\left(T_{s_{2}+i, d_{2}+i}\right) \geq m_{k}\left(T_{d_{2}+i+2, d_{2}+i}\right) \geq m_{k}\left(T_{d+1, d-1}\right) \geq m_{k}\left(T_{d+1, d-2}\right) .
\end{aligned}
$$

When $p=1$. If $v=v_{i}$, then $i \geq 1$ and $H \supseteq P_{i} \cup G_{3}$, where $G_{3} \in$ $\mathcal{U}\left(s_{3}, d_{3}\right), d_{3} \geq d-i \geq 3, s_{3} \geq d_{3}+2$. Accordingly,

$$
\begin{aligned}
m_{k}(H) & \geq m_{k}\left(P_{i} \cup G_{3}\right) \geq m_{k}\left(P_{i} \cup U_{s_{3}, d_{3}}\right) \geq m_{k}\left(P_{i} \cup T_{s_{3}, d_{3}}\right) \geq m_{k}\left(T_{s_{3}+i-1, d_{3}+i-1}\right) \\
& \geq m_{k}\left(T_{d_{2}+i+2, d_{2}+i}\right) \geq m_{k}\left(T_{d+1, d-1}\right) \geq m_{k}\left(T_{d+1, d-2}\right) .
\end{aligned}
$$

If $v=v_{i+1}$, then $H \supseteq P_{i+2} \cup G_{4}$, where $G_{4} \in \mathcal{U}\left(s_{4}, d_{4}\right), d_{4} \geq d-(i+1)-1=$ $d-i-2 \geq 1, s_{4} \geq d_{4}+2$.

If $d_{4}=1$, then $i=d-3, G_{4}=C_{3}$, we have

$$
m_{k}(H) \geq m_{k}\left(P_{d-1} \cup C_{3}\right) \geq m_{k}\left(P_{d-1} \cup P_{3}\right) \geq m_{k}\left(P_{d+1}\right) \geq m_{k}\left(T_{d+1, d-2}\right) .
$$

If $d_{4}=2$, then $s_{4} \geq 4, i \geq d-4$. Consequently,

$$
\begin{aligned}
m_{k}(H) & \geq m_{k}\left(P_{i+2} \cup G_{4}\right) \geq m_{k}\left(P_{i+2} \cup S_{s_{4}}\right) \geq m_{k}\left(P_{i+2} \cup T_{4,2}\right) \\
& \geq m_{k}\left(T_{i+5, i+3}\right) \geq m_{k}\left(T_{d+1, d-1}\right) \geq m_{k}\left(T_{d+1, d-2}\right) .
\end{aligned}
$$

If $d_{4} \geq 3$. Then

$$
\begin{aligned}
m_{k}(H) & \geq m_{k}\left(P_{i+2} \cup G_{4}\right) \geq m_{k}\left(P_{i+2} \cup U_{s_{4}, d_{4}}\right) \geq m_{k}\left(P_{i+2} \cup T_{s_{4}, d_{4}}\right) \\
& \geq m_{k}\left(T_{s_{4}+i+1, d_{4}+i+1}\right) \geq m_{k}\left(T_{d+1, d-1}\right) \geq m_{k}\left(T_{d+1, d-2}\right) .
\end{aligned}
$$


Now suppose that $p \geq 2$. If $v \neq x_{i}, x_{i+p}$, then $H \supseteq G_{5}$, where $G_{5} \in$ $\mathcal{U}\left(s_{5}, d_{5}\right), d_{5} \geq d, s_{5} \geq d_{5}+2$. Hence

$$
m_{k}(H) \geq m_{k}\left(G_{5}\right) \geq m_{k}\left(U_{s_{5}, d_{5}}\right) \geq m_{k}\left(T_{s_{5}, d_{5}}\right) \geq m_{k}\left(T_{d+1, d-2}\right) .
$$

If $v=x_{i}$, then $H \supseteq P_{i} \cup G_{6}$, where $G_{6} \in \mathcal{U}\left(s_{6}, d_{6}\right), d_{6} \geq d-i-1 \geq 3, s_{6} \geq$ $d_{6}+3$. Therefore,

$$
\begin{aligned}
m_{k}(H) & \geq m_{k}\left(P_{i} \cup G_{6}\right) \geq m_{k}\left(P_{i} \cup U_{s_{6}, d_{6}}\right) \geq m_{k}\left(P_{i} \cup T_{s_{6}, d_{6}}\right) \\
& \geq m_{k}\left(T_{s_{6}+i-1, d_{6}+i-1}\right) \geq m_{k}\left(T_{d+1, d-2}\right) .
\end{aligned}
$$

If $v=x_{i+p}$, then $H \supseteq T_{1} \cup G_{7}$ or $P_{i+p+1} \cup G_{7}$, where $G_{7} \in \mathcal{U}\left(s_{7}, d_{7}\right), d_{7} \geq$ $d-i-p-1 \geq 1, s_{7} \geq d_{7}+2, T_{1} \in \mathcal{T}\left(i+p+1, d_{T_{1}}\right), d_{T_{1}} \geq i+p-1$. If $d_{7}=1$, then $i+p=d-2, G_{7}=C_{3}$, thus

$$
m_{k}(H) \geq m_{k}\left(T_{d-1, d-3} \cup C_{3}\right) \geq m_{k}\left(T_{d-1, d-3} \cup P_{3}\right) \geq m_{k}\left(T_{d+1, d-2}\right)
$$

or

$$
\begin{aligned}
m_{k}(H) & \geq m_{k}\left(P_{i+p+1} \cup G_{7}\right) \geq m_{k}\left(P_{i+p+1} \cup C_{3}\right) \geq m_{k}\left(P_{i+p+1} \cup P_{3}\right) \\
& \geq m_{k}\left(P_{i+p+3}\right)=m_{k}\left(P_{d+1}\right) \geq m_{k}\left(T_{d+1, d-2}\right) .
\end{aligned}
$$

If $d_{7}=2$, then $i+p \geq d-3, s_{7} \geq 4$, accordingly,

$$
\begin{aligned}
m_{k}(H) & \geq m_{k}\left(T_{i+p+1, i+p-1} \cup S_{s_{7}}\right) \geq m_{k}\left(T_{i+p+1, i+p-1} \cup T_{4,2}\right) \\
& \geq m_{k}\left(T_{i+p+4, i+p+1}\right) \geq m_{k}\left(T_{d+1, d-2}\right)
\end{aligned}
$$

or

$m_{k}(H) \geq m_{k}\left(P_{i+p+1} \cup S_{s_{7}}\right) \geq m_{k}\left(P_{i+p+1} \cup T_{4,2}\right) \geq m_{k}\left(T_{i+p+4, i+p+2}\right) \geq m_{k}\left(T_{d+1, d-2}\right)$.

If $d_{7} \geq 3$, then

$$
\begin{aligned}
m_{k}(H) & \geq m_{k}\left(T_{i+p+1, i+p-1} \cup U_{s_{7}, d_{7}}\right) \geq m_{k}\left(T_{i+p+1, i+p-1} \cup T_{s_{7}, d_{7}}\right) \\
& \geq m_{k}\left(T_{i+p+s_{7}, i+p+d_{7}-1}\right) \geq m_{k}\left(T_{d+1, d-2}\right)
\end{aligned}
$$

or

$m_{k}(H) \geq m_{k}\left(P_{i+p+1} \cup U_{s_{7}, d_{7}}\right) \geq m_{k}\left(P_{i+p+1} \cup T_{s_{7}, d_{7}}\right) \geq m_{k}\left(T_{i+p+s_{7}, i+p+d_{7}}\right) \geq m_{k}\left(T_{d+1, d-2}\right)$.

Subcase $2.2 v$ lies outside any cycle. Then $H \supseteq C_{a} \cup C_{b}$.

First, suppose that $v$ lies on $P(G)$ and take $v=x_{i}$. If $P(G)$ has no common vertices with any cycle, then $H \supseteq C_{a} \cup C_{b} \cup P_{i} \cup P_{d-i}$. Thus $m_{k}(H) \geq m_{k}\left(C_{a} \cup C_{b} \cup P_{i} \cup P_{d-i}\right) \geq m_{k}\left(P_{3} \cup P_{d-1}\right) \geq m_{k}\left(P_{d+1}\right) \geq m_{k}\left(T_{d+1, d-2}\right)$. 
If $P(G)$ has no common vertices with exactly one cycle, say $C_{a}$. Then $H \supseteq C_{a} \cup P_{i} \cup G_{1}$, where $G_{1} \in \mathcal{U}\left(s_{1}, d_{1}\right), d_{1} \geq d-i-1, s_{1} \geq d_{1}+2$. If $d_{1}=1$, then $i=d-2, G_{1}=C_{3}$, hence

$m_{k}(H) \geq m_{k}\left(C_{a} \cup P_{i} \cup C_{3}\right) \geq m_{k}\left(P_{2} \cup P_{i} \cup P_{3}\right) \geq m_{k}\left(P_{i+3}\right)=m_{k}\left(P_{d+1}\right) \geq m_{k}\left(T_{d+1, d-2}\right)$.

If $d_{1}=2$, then $i \geq d-3, s_{1} \geq 4$. Consequently,

$m_{k}(H) \geq m_{k}\left(C_{a} \cup P_{i} \cup G_{1}\right) \geq m_{k}\left(P_{2} \cup P_{i} \cup T_{s_{1}, 2}\right) \geq m_{k}\left(T_{i+s_{1}, i+2}\right) \geq m_{k}\left(T_{d+1, d-2}\right)$.

If $d_{1} \geq 3$, then

$$
\begin{aligned}
m_{k}(H) & \geq m_{k}\left(C_{a} \cup P_{i} \cup G_{1}\right) \geq m_{k}\left(P_{2} \cup P_{i} \cup U_{s_{1}, d_{1}}\right) \geq m_{k}\left(P_{2} \cup P_{i} \cup T_{s_{1}, d_{1}}\right) \\
& \geq m_{k}\left(P_{i+1} \cup T_{s_{1}, d_{1}}\right) \geq m_{k}\left(T_{s_{1}+i, d_{1}+i}\right) \geq m_{k}\left(T_{d+1, d-2}\right) .
\end{aligned}
$$

If $P(G)$ has common vertices with both cycles, then $H \supseteq P_{i} \cup G_{2}$ or $G_{3} \cup G_{4}$, where $G_{2} \in \mathcal{U}\left(s_{2}, d_{2}\right), G_{3} \in \mathcal{U}\left(s_{3}, d_{3}\right), G_{4} \in \mathcal{U}\left(s_{4}, d_{4}\right)$. Meanwhile, $d_{2} \geq$ $d-i-1 \geq 3, n-2-i \geq s_{2} \geq d_{2}+3, d_{3} \geq i-1 \geq 1, s_{3} \geq d_{3}+2$, $d_{4} \geq d-i-1 \geq 1, s_{4} \geq d_{4}+2$.

Suppose that $H \supseteq P_{i} \cup G_{2}$. Since $s_{2}-d_{2} \leq n-2-i-(d-i-1)=n-d-1<h$, thus by the induction hypothesis, $G_{2} \succ B_{s_{2}, d_{2}}$. Then

$$
\begin{aligned}
m_{k}(H) & \geq m_{k}\left(P_{i} \cup G_{2}\right) \geq m_{k}\left(P_{i} \cup B_{s_{2}, d_{2}}\right) \geq m_{k}\left(P_{i} \cup T_{s_{2}, d_{2}}\right) \\
& \geq m_{k}\left(T_{s_{2}+i-1, d_{2}+i-1}\right) \geq m_{k}\left(T_{d+1, d-2}\right) .
\end{aligned}
$$

Suppose that $H \supseteq G_{3} \cup G_{4}$. If $d_{3}=d_{4}=1$, then $n=8, d=4, G_{3}=G_{4}=$ $C_{3}$. In this case, it is easy to obtain that $G \succ B_{8,4}$.

If $d_{3}=2, d_{4}=1$, then $d=5, s_{3} \geq 4, G_{4}=C_{3}$. We can have

$m_{k}(H) \geq m_{k}\left(G_{3} \cup G_{4}\right) \geq m_{k}\left(S_{s_{3}} \cup C_{3}\right) \geq m_{k}\left(T_{4,2} \cup P_{3}\right) \geq m_{k}\left(T_{6,4}\right) \geq m_{k}\left(T_{d+1, d-2}\right)$.

If $d_{3} \geq 3, d_{4}=1$, then $d_{3} \geq d-3$. Accordingly,

$$
\begin{aligned}
m_{k}(H) & \geq m_{k}\left(G_{3} \cup G_{4}\right) \geq m_{k}\left(U_{s_{3}, d_{3}} \cup C_{3}\right) \geq m_{k}\left(T_{s_{3}, d_{3}} \cup P_{3}\right) \\
& \geq m_{k}\left(T_{s_{3}+2, d_{3}+2}\right) \geq m_{k}\left(T_{d+1, d-2}\right) .
\end{aligned}
$$

If $d_{3}=2, d_{4}=2$, then $d=6, s_{3} \geq 4, s_{4} \geq 4$. Hence

$m_{k}(H) \geq m_{k}\left(G_{3} \cup G_{4}\right) \geq m_{k}\left(S_{s_{3}} \cup S_{s_{4}}\right) \geq m_{k}\left(T_{4,2} \cup T_{4,2}\right) \geq m_{k}\left(T_{7,4}\right)=m_{k}\left(T_{d+1, d-2}\right)$.

If $d_{3} \geq 3, d_{4}=2$, then $d_{3} \geq d-4, s_{4} \geq 4$. Thus

$$
\begin{aligned}
m_{k}(H) & \geq m_{k}\left(G_{3} \cup G_{4}\right) \geq m_{k}\left(U_{s_{3}, d_{3}} \cup S_{s_{4}}\right) \geq m_{k}\left(T_{s_{3}, d_{3}} \cup T_{s_{4}, 2}\right) \\
& \geq m_{k}\left(T_{s_{3}+s_{4}-1, d_{3}+2}\right) \geq m_{k}\left(T_{d+1, d-2}\right) .
\end{aligned}
$$

If $d_{3} \geq 3, d_{4} \geq 3$, then $d_{3}+d_{4} \geq d-2$. Therefore,

$$
\begin{aligned}
m_{k}(H) & \geq m_{k}\left(G_{3} \cup G_{4}\right) \geq m_{k}\left(U_{s_{3}, d_{3}} \cup U_{s_{4}, d_{4}}\right) \geq m_{k}\left(T_{s_{3}, d_{3}} \cup T_{s_{4}, d_{4}}\right) \\
& \geq m_{k}\left(T_{s_{3}+s_{4}-1, d_{3}+d_{4}}\right) \geq m_{k}\left(T_{d+1, d-2}\right) .
\end{aligned}
$$


Next, suppose that $v$ lies outside $P(G)$. Then $H \supseteq C_{a} \cup C_{b} \cup P(G)$, $C_{a} \cup G_{5}$ or $G_{6}$, where $G_{5} \in \mathcal{U}\left(s_{5}, d\right)$ with $s_{5} \geq d+2$ and $G_{6} \in \mathcal{B}\left(s_{6}, d\right)$ with $d+3 \leq s_{6} \leq n-2$. It is easy to show as above that $m_{k}(H) \geq m_{k}\left(T_{d+1, d-2}\right)$.

Case 3 Any diametrical path of $G$ contains all pendent vertices.

Let $P(G)=x_{0} x_{1} \cdots x_{d}$ be any diametrical path of $G$. Suppose that $y_{0} y_{1} \cdots y_{p}$ is a path whose internal vertices $y_{1}, y_{2}, \ldots, y_{p-1}$ all have degree two and $y_{p}$ is a pendent vertex. Then we call it a pendent path, denoted by $\left(y_{0}, y_{p}\right)$.

Subcase 3.1 There are exactly two pendent vertices in $G$, namely, $x_{0}$ and $x_{d}$.

Suppose that $\operatorname{deg}_{G}\left(x_{i}\right), \operatorname{deg}_{G}\left(x_{l}\right) \geq 3$ such that $\left(x_{i}, x_{0}\right)$ and $\left(x_{l}, x_{d}\right)$ are distinct pendent paths. Let $s=l-i$.

If $s=0$, i.e., $x_{i}=x_{l}$. Then $i \geq 3, l \leq d-3$. Since

$$
\begin{aligned}
m_{k}(G)= & m_{k}\left(G-x_{i-3} x_{i-2}\right)+m_{k-1}\left(G-x_{i-3}-x_{i-2}\right) \\
= & m_{k}\left(G-x_{i-3} x_{i-2}-x_{l+1} x_{l+2}\right)+m_{k-1}\left(G-x_{i-3} x_{i-2}-x_{l+1}-x_{l+2}\right) \\
& +m_{k-1}\left(G-x_{i-3}-x_{i-2}-x_{l+2} x_{l+3}\right)+m_{k-2}\left(G-x_{i-3}-x_{i-2}-x_{l+2}-x_{l+3}\right) \\
= & m_{k}\left(G_{1} \cup P_{i-2} \cup P_{d-i-1}\right)+m_{k-1}\left(G_{3} \cup P_{i-2} \cup P_{d-i-2}\right) \\
& +m_{k-1}\left(G_{2} \cup P_{i-3} \cup P_{d-i-2}\right)+m_{k-2}\left(G_{4} \cup P_{i-3} \cup P_{d-i-3}\right)
\end{aligned}
$$

and

$$
\begin{aligned}
m_{k}\left(B_{n, d}\right)= & m_{k}\left(B_{n-d+3,3} \cup P_{d-3}\right)+m_{k-1}\left(S_{n-d+2} \cup P_{d-4}\right) \\
= & m_{k}\left(B_{n-d+3,3} \cup P_{i-2} \cup P_{d-i-1}\right)+m_{k-1}\left(B_{n-d+3,3} \cup P_{i-3} \cup P_{d-i-2}\right) \\
& +m_{k-1}\left(S_{n-d+2} \cup P_{i-2} \cup P_{d-i-2}\right)+m_{k-2}\left(S_{n-d+2} \cup P_{i-3} \cup P_{d-i-3}\right),
\end{aligned}
$$

it suffices to prove that $G_{1}, G_{2} \succ B_{n-d+3,3}$ and $G_{3}, G_{4} \succ S_{n-d+2}$, where $G_{1}=G-\left(x_{i-3}, x_{0}\right)-\left(x_{l+2}, x_{d}\right) \in \mathcal{B}\left(n-d+3, d_{1}\right), G_{2}=G-\left(x_{i-2}, x_{0}\right)-$ $\left(x_{l+3}, x_{d}\right) \in \mathcal{B}\left(n-d+3, d_{2}\right), G_{3}=G-\left(x_{i-3}, x_{0}\right)-\left(x_{l+1}, x_{d}\right), G_{4}=G-$ $\left(x_{i-2}, x_{0}\right)-\left(x_{l+2}, x_{d}\right), d_{1} \geq 4, d_{2} \geq 4$. Since $n-d+3-d_{1} \leq n-d-1<h$, $n-d+3-d_{2} \leq n-d-1<h$. Then by the induction hypothesis, $G_{1} \succ$ $B_{n-d+3, d_{1}} \succ B_{n-d+3,3}, G_{2} \succ B_{n-d+3, d_{2}} \succ B_{n-d+3,3}$. In addition, both $G_{3}$ and $G_{4}$ are bicyclic graphs with $n-d+2$ vertices, consequently, we have $G_{3}, G_{4} \succ S_{n-d+2}$.

If $s=1$ or $s=2$, then by similar arguments as above, we have the desired result.

If $s \geq 3$, it is easy to obtain that $i \geq 2$ and $l \leq d-2$. Then

$$
\begin{aligned}
m_{k}(G)= & m_{k}\left(G-x_{i-2} x_{i-1}\right)+m_{k-1}\left(G-x_{i-2}-x_{i-1}\right) \\
= & m_{k}\left(G-x_{i-2} x_{i-1}-x_{l+1} x_{l+2}\right)+m_{k-1}\left(G-x_{i-2} x_{i-1}-x_{l+1}-x_{l+2}\right) \\
& +m_{k-1}\left(G-x_{i-2}-x_{i-1}-x_{l+1} x_{l+2}\right)+m_{k-2}\left(G-x_{i-2}-x_{i-1}-x_{l+1}-x_{l+2}\right) \\
= & m_{k}\left(G_{5} \cup P_{i-1} \cup P_{d-l-1}\right)+m_{k-1}\left(G_{7} \cup P_{i-1} \cup P_{d-l-2}\right) \\
& +m_{k-1}\left(G_{6} \cup P_{i-2} \cup P_{d-l-1}\right)+m_{k-2}\left(G_{8} \cup P_{i-2} \cup P_{d-l-2}\right),
\end{aligned}
$$




$$
\begin{aligned}
m_{k}\left(B_{n, d}\right)= & m_{k}\left(B_{n-d+l+1, l+1} \cup P_{d-l-1}\right)+m_{k-1}\left(B_{n-d+l, l} \cup P_{d-l-2}\right) \\
= & m_{k}\left(B_{n-d+s+2, s+2} \cup P_{i-1} \cup P_{d-l-1}\right)+m_{k-1}\left(B_{n-d+s+1, s+1} \cup P_{i-2} \cup P_{d-l-1}\right) \\
& +m_{k-1}\left(B_{n-d+s+1, s+1} \cup P_{i-1} \cup P_{d-l-2}\right)+m_{k-2}\left(B_{n-d+s, s} \cup P_{i-2} \cup P_{d-l-2}\right) .
\end{aligned}
$$

Hence it suffices to show that $G_{5} \succ B_{n-d+s+2, s+2}, G_{6}, G_{7} \succ B_{n-d+s+1, s+1}$, $G_{8} \succ B_{n-d+s, s}$.

Let $d_{j}=d\left(G_{j}\right)$, and $n_{j}=\left|V\left(G_{j}\right)\right|$, where $j=5,6,7,8$. Then $d_{j} \geq 4$. If $n_{j}-d_{j}<h$ holds for all $j \in\{5,6,7,8\}$, then by the induction hypothesis and previous Lemmas, we have the desired results. Otherwise, there exists at least a $j \in\{5,6,7,8\}$ such that $n_{j}-d_{j}=h$. When $j=5, G_{5} \in$ $\mathcal{B}(n-d+s+2, s+2)$. If there exists some diametrical path $P\left(G_{5}\right)$ such that $x_{i-1}$ or $x_{l+1}$ lies outside $P\left(G_{5}\right)$, the proof is similar with Case 2, thus $G_{5} \succ B_{n-d+s+2, s+2}$. Otherwise, $G_{5}-x_{i-1} \in \mathcal{B}(n-d+s+1, s+1)$, then by Lemma 4.3, $G_{5} \succ B_{n-d+s+1, s+2}$. We also have $G_{5}-x_{i-1}-x_{i} \succeq U_{n-d+s, s} \succ$ $T_{n-d+s, s} \succ T_{s+3, s}$. Therefore, $G_{5} \succ B_{n-d+s+2, s+2}$.

When $j=7, G_{7} \in \mathcal{B}(n-d+s+1, s+1)$. If $x_{i-1}$ lies on all diametrical paths of $G_{7}$, then by Lemma 4.3, $G_{7} \succ B_{n-d+s+1, s+2} \succ B_{n-d+s+1, s+1}$. Otherwise, in the same way as in Case 2, we can also obtain $G_{7} \succ B_{n-d+s+1, s+1}$.

Similarly, when $j=6$, we can have $G_{6} \succ B_{n-d+s+1, s+1}$.

When $j=8, G_{8} \in \mathcal{B}(n-d+s, s)$. Since $G_{8}$ contains no pendent vertices, then by Lemmas 2.11 and $4.2, G_{8} \succ B_{n-d+s, s+1} \succ B_{n-d+s, s}$.

Subcase 3.2 There is only one pendent vertex in $G$, say $x_{0}$.

Since there are two vertex-disjoint cycles in $G, \operatorname{deg}_{G}\left(x_{d}\right)=2$. Suppose that $x_{l}$ is the vertex such that $\operatorname{deg}_{G}\left(x_{l}\right) \geq 3$ and $\operatorname{deg}_{G}\left(x_{i}\right)=2$ for $l+1 \leq$ $i \leq d$. It is easy to check that $l \leq d-2$. Then $G-x_{d-1} x_{d} \in \mathcal{U}\left(n, d_{9}\right)$, where $d_{9} \geq d$, and $G-x_{d-1}-x_{d} \in \mathcal{U}\left(n-2, d_{10}\right)$, where $d_{10} \geq d-1$. Hence,

$$
\begin{aligned}
m_{k}(G) & =m_{k}\left(G-x_{d-1} x_{d}\right)+m_{k-1}\left(G-x_{d-1}-x_{d}\right) \\
& \geq m_{k}\left(U_{n, d_{9}}\right)+m_{k-1}\left(U_{n-2, d_{10}}\right) \\
& \geq m_{k}\left(U_{n, d}\right)+m_{k-1}\left(U_{n-2, d-1}\right) \\
& \geq m_{k}\left(U_{n, d}\right)+m_{k-1}\left(T_{n-2, d-1}\right) \\
& \geq m_{k}\left(U_{n, d}\right)+m_{k-1}\left(P_{d-3} \cup S_{n-d+1}\right) \\
& =m_{k}\left(B_{n, d}\right) .
\end{aligned}
$$

In particular, $m_{2}(G)>m_{2}\left(B_{n, d}\right)$. Thus, $G \succ B_{n, d}$.

Therefore, we complete the proof.

Theorem 4.5 Let $G \in \mathcal{B}(n, d)$ with $n \geq 8,3 \leq d \leq n-3$ and $G \neq B_{n, d}$. If there is no vertex-disjoint cycles in $G$, then $G \succ B_{n, d}$.

Proof. We will prove this theorem by induction on $n-d$.

By Lemma 4.1, the result holds for $n-d=3$. Let $h \geq 4$ and suppose that the result holds for $n-d<h$. Now assume that $n-d=h$, let $G \in \mathcal{B}(n, d)$ and $G \neq B_{n, d}$. 
Case 1 If $G$ contains no pendent vertices.

Then by Lemmas 2.11 and $4.2, G \succ B_{n, d+1} \succ B_{n, d}$.

Case 2 If there exists a pendent vertex outside some diametrical path $P(G)=x_{0} x_{1} \ldots x_{d}$.

Let $u$ be a pendent vertex outside $P(G)$ and $v$ be its unique neighbor. Then $G-u \in \mathcal{B}(n-1, d)$. If $G-u=B_{n-1, d}$, then it can be checked that $G-u-v \succ T_{d+1, d-2}$. And thus from (*), we can obtain that $G \succ B_{n, d}$. Otherwise, by the induction hypothesis, we have $G-u \succ B_{n-1, d}$. Let $H=G-u-v$, in order to prove the result, we only need to show that $m_{k}(H) \geq m_{k}\left(T_{d+1, d-2}\right)$. We choose $C_{a}, C_{b}$ as above in $G$, and if there exists the third cycle, denote it by $C_{c}$.

Subcase 2.1 When $v$ lies on some cycle, say $C_{a}$.

First, suppose that $v=u_{0}$ or $u_{t}$, then $H$ contains no cycles. If $v$ lies outside $P(G)$, then $H \supseteq P(G)$. Thus $m_{k}(H) \geq m_{k}(P(G))=m_{k}\left(P_{d+1}\right) \geq$ $m_{k}\left(T_{d+1, d-2}\right)$. If $v$ lies on $P(G)$, say $v=x_{i}$.

(1) If $C_{a}$ and $C_{b}$ have exactly one common vertex, then $H \supseteq P_{2} \cup P_{2} \cup$ $P_{i} \cup P_{d-i}, P_{2} \cup P_{i} \cup P_{d-i+1}, P_{2} \cup P_{i+1} \cup P_{d-i}, P_{i+1} \cup P_{d-i+1}, P_{2} \cup P_{i} \cup T_{1}$, $P_{2} \cup P_{d-i} \cup T_{2}, P_{i+1} \cup T_{1}, P_{d-i+1} \cup T_{2}$ or $T_{1} \cup T_{2}$, where $T_{1} \in \mathcal{T}(d-i+1, d-i-1)$ and $T_{2} \in \mathcal{T}(i+1, i-1)$.

If $H \supseteq P_{2} \cup P_{2} \cup P_{i} \cup P_{d-i}, P_{2} \cup P_{i} \cup P_{d-i+1}, P_{2} \cup P_{i+1} \cup P_{d-i}$ or $P_{i+1} \cup P_{d-i+1}$, then $m_{k}(H) \geq m_{k}\left(P_{d+1}\right) \geq m_{k}\left(T_{d+1, d-2}\right)$;

If $H \supseteq P_{2} \cup P_{i} \cup T_{1}$, then $m_{k}(H) \geq m_{k}\left(P_{i+1} \cup T_{1}\right) \geq m_{k}\left(P_{i+1} \cup\right.$ $\left.T_{d-i+1, d-i-1}\right) \geq m_{k}\left(T_{d+1, d-1}\right) \geq m_{k}\left(T_{d+1, d-2}\right)$; Similarly, if $H \supseteq P_{2} \cup P_{d-i} \cup$ $T_{2}$, we also have $m_{k}(H) \geq m_{k}\left(T_{d+1, d-2}\right)$;

If $H \supseteq T_{1} \cup T_{2}$, then $m_{k}(H) \geq m_{k}\left(T_{1} \cup T_{2}\right) \geq m_{k}\left(T_{d-i+1, d-i-1} \cup\right.$ $\left.T_{i+1, i-1}\right) \geq m_{k}\left(T_{d+1, d-2}\right)$;

If $H \supseteq P_{i+1} \cup T_{1}$ or $H \supseteq P_{d-i+1} \cup T_{2}$, then $m_{k}(H) \geq m_{k}\left(T_{1} \cup T_{2}\right) \geq$ $m_{k}\left(T_{d+1, d-2}\right)$.

(2) If $C_{a}$ and $C_{b}$ have at least two common vertices, then $H \supseteq P_{3} \cup P_{i} \cup$ $P_{d-i}, P_{i} \cup P_{d-i+2}, P(G), P_{i} \cup T_{3}$ or $P_{i} \cup T_{4}$, where $T_{3} \in \mathcal{T}(d-i+2, d-i-1)$ and $T_{4} \in \mathcal{T}(d-i+2, d-i)$.

If $H \supseteq P_{3} \cup P_{i} \cup P_{d-i}, P_{i} \cup P_{d-i+2}$ or $P(G)$, then $m_{k}(H) \geq m_{k}\left(P_{d+1}\right) \geq$ $m_{k}\left(T_{d+1, d-2}\right)$;

If $H \supseteq P_{i} \cup T_{3}$, then $m_{k}(H) \geq m_{k}\left(P_{i} \cup T_{3}\right) \geq m_{k}\left(P_{i} \cup T_{d-i+2, d-i-1}\right) \geq$ $m_{k}\left(T_{d+1, d-2}\right)$;

If $H \supseteq P_{i} \cup T_{4}$, then $m_{k}(H) \geq m_{k}\left(P_{i} \cup T_{4}\right) \geq m_{k}\left(P_{i} \cup T_{d-i+2, d-i}\right) \geq$ $m_{k}\left(T_{d+1, d-1}\right) \geq m_{k}\left(T_{d+1, d-2}\right)$.

Next, suppose that $v \neq u_{0}$ and $v \neq u_{t}$. If $v$ lies outside $P(G)$, then $H \supseteq P(G)$, similarly, we have $m_{k}(H) \geq m_{k}\left(T_{d+1, d-2}\right)$. So suppose that $v$ lies on $P(G)$. Then $P(G)$ and $C_{a}$ have common vertices, say $x_{i}, \ldots, x_{i+p}$, where $p \geq 0$.

(1) If $p=0$, then $i \geq 1$ and $H \supseteq P_{i} \cup P_{d-i} \cup C_{s}$, where $s=b$ or $c$. It 
follows that

$$
\begin{aligned}
m_{k}(H) & \geq m_{k}\left(P_{i} \cup P_{d-i} \cup C_{s}\right) \geq m_{k}\left(P_{i} \cup P_{d-i} \cup S_{s}\right) \geq m_{k}\left(P_{i} \cup P_{d-i} \cup P_{3}\right) \\
& \geq m_{k}\left(P_{d+1}\right) \geq m_{k}\left(T_{d+1, d-2}\right) .
\end{aligned}
$$

(2) If $p \geq 1$. When $v \neq x_{i}$ and $v \neq x_{i+p}$, then $H \supseteq G_{1}$, where $G_{1} \in$ $\mathcal{U}\left(s_{1}, d_{1}\right), d_{1} \geq d$ and $s_{1} \geq d_{1}+2$. Thus

$$
m_{k}(H) \geq m_{k}\left(G_{1}\right) \geq m_{k}\left(U_{s_{1}, d_{1}}\right) \geq m_{k}\left(T_{s_{1}, d_{1}}\right) \geq m_{k}\left(T_{s_{1}, d}\right) \geq m_{k}\left(T_{d+1, d-2}\right) .
$$

When $v=x_{i}$ or $x_{i+p}$, say $v=x_{i}(i \geq 1)$, then $H \supseteq P_{i} \cup G_{2}$, where $G_{2} \in$ $\mathcal{U}\left(s_{2}, d_{2}\right), d_{2} \geq d-i-1 \geq 2\left(d-i-1 \geq 1\right.$, for $d-i-1=1$, clearly $m_{k}(H) \geq$ $\left.m_{k}\left(T_{i+3, i}\right)\right)$ and $s_{2} \geq d-i+2$; or $H \supseteq P_{i} \cup G_{3}$, where $G_{3} \in \mathcal{U}\left(s_{3}, d_{3}\right)$, $d_{3} \geq d-i \geq 2$ and $s_{3} \geq d_{3}+2$; or $H \supseteq P_{i} \cup G^{\prime}$, where $G^{\prime}$ is the graph obtained by attaching a path $P_{d-i-2}$ to a vertex of $C_{b}=C_{3}$.

Suppose that $H \supseteq P_{i} \cup G_{2}$. If $d_{2}=2$, then $i=d-3$ and $s_{2} \geq 5$. Hence,

$m_{k}(H) \geq m_{k}\left(P_{i} \cup G_{2}\right) \geq m_{k}\left(P_{i} \cup S_{s_{2}}\right) \geq m_{k}\left(P_{i} \cup T_{5,2}\right) \geq m_{k}\left(T_{i+4, i+1}\right)=m_{k}\left(T_{d+1, d-2}\right)$.

If $d_{2} \geq 3$, then

$$
\begin{aligned}
m_{k}(H) & \geq m_{k}\left(P_{i} \cup G_{2}\right) \geq m_{k}\left(P_{i} \cup U_{s_{2}, d_{2}}\right) \geq m_{k}\left(P_{i} \cup T_{s_{2}, d_{2}}\right) \\
& \geq m_{k}\left(T_{i+s_{2}-1, i+d_{2}-1}\right) \geq m_{k}\left(T_{d+1, d-1}\right) \geq m_{k}\left(T_{d+1, d-2}\right) .
\end{aligned}
$$

Suppose that $H \supseteq P_{i} \cup G_{3}$. If $d_{3}=2$, then $i=d-2$ and $s_{3} \geq 4$. Thus

$$
\begin{aligned}
m_{k}(H) & \geq m_{k}\left(P_{i} \cup G_{3}\right) \geq m_{k}\left(P_{i} \cup S_{s_{3}}\right) \geq m_{k}\left(P_{i} \cup T_{4,2}\right) \\
& \geq m_{k}\left(T_{d+1, d-1}\right) \geq m_{k}\left(T_{d+1, d-2}\right) .
\end{aligned}
$$

If $d_{3} \geq 3$, then

$$
\begin{aligned}
m_{k}(H) & \geq m_{k}\left(P_{i} \cup G_{3}\right) \geq m_{k}\left(P_{i} \cup U_{s_{3}, d_{3}}\right) \geq m_{k}\left(P_{i} \cup T_{s_{3}, d_{3}}\right) \\
& \geq m_{k}\left(T_{i+s_{3}-1, i+d_{3}-1}\right) \geq m_{k}\left(T_{d+1, d-1}\right) \geq m_{k}\left(T_{d+1, d-2}\right) .
\end{aligned}
$$

Suppose that $H \supseteq P_{i} \cup G^{\prime}$. If $d-i-2=0$, then $i=d-2$ and $H \supseteq P_{i} \cup C_{3}$. Therefore,

$m_{k}(H) \geq m_{k}\left(P_{i} \cup G^{\prime}\right) \geq m_{k}\left(P_{i} \cup T_{4,2}\right) \geq m_{k}\left(T_{i+3, i+1}\right)=m_{k}\left(T_{d+1, d-1}\right) \geq m_{k}\left(T_{d+1, d-2}\right)$.

If $d-i-2 \geq 1$, choose an edge $u_{0} u_{1}$ of $G^{\prime}$ in $C_{3}$ such that both $u_{0}$ and $u_{1}$ have degree 2 . Then

$$
\begin{aligned}
m_{k}(H) & \geq m_{k}\left(P_{i} \cup G^{\prime}\right) \\
& =m_{k}\left(P_{i} \cup G^{\prime}-u_{0} u_{1}\right)+m_{k-1}\left(P_{i} \cup G^{\prime}-u_{0}-u_{1}\right) \\
& =m_{k}\left(P_{i} \cup T_{d-i+1, d-i-1}\right)+m_{k-1}\left(P_{i} \cup P_{d-i-1}\right) \\
& \geq m_{k}\left(T_{d, d-2}\right)+m_{k-1}\left(P_{d-3}\right) \\
& =m_{k}\left(T_{d+1, d-2}\right) .
\end{aligned}
$$


Subcase 2.2 When $v$ lies outside any cycle.

In this case, $H$ contains two cycles $C_{a}$ and $C_{b}$ with at least one common vertex. Let $C_{a} \cdot C_{b}$ denote the subgraph of $G$ induced by $V\left(C_{a}\right) \cup V\left(C_{b}\right)$.

First, suppose that $v$ lies on $P(G)$, say $v=x_{i}$. Thus,

If vertices on $P(G)$ lie outside any cycle, then $H \supseteq C_{a} \cdot C_{b} \cup P_{i} \cup P_{d-i}$.

$m_{k}(H) \geq m_{k}\left(C_{a} \cdot C_{b} \cup P_{i} \cup P_{d-i}\right) \geq m_{k}\left(P_{3} \cup P_{i} \cup P_{d-i}\right) \geq m_{k}\left(P_{d+1}\right) \geq m_{k}\left(T_{d+1, d-2}\right)$.

Otherwise, $H \supseteq P_{i} \cup G_{1}$, where $G_{1} \in \mathcal{B}\left(s_{1}, d_{1}\right), d_{1} \geq \max \{d-i-1,2\}$ and $d_{1}+2 \leq s_{1} \leq n-2-i$.

Suppose that $s_{1} \geq d_{1}+3$. If $d_{1}=2$, then $i \geq d-3$ and $s_{1} \geq 5$. Thus we have

$m_{k}(H) \geq m_{k}\left(P_{i} \cup G_{1}\right) \geq m_{k}\left(P_{i} \cup S_{s_{1}}\right) \geq m_{k}\left(P_{i} \cup T_{5,2}\right) \geq m_{k}\left(T_{i+4, i+1}\right) \geq m_{k}\left(T_{d+1, d-2}\right)$.

If $d_{1} \geq 3$, then $d-i-1 \leq d_{1}$, which deduces that $s_{1}-d_{1} \leq n-2-i-(d-$ $i-1)=n-d-1<h$. By the induction hypothesis, $G_{1} \succ B_{s_{1}, d_{1}}$, therefore,

$m_{k}(H) \geq m_{k}\left(P_{i} \cup G_{1}\right) \geq m_{k}\left(P_{i} \cup B_{s_{1}, d_{1}}\right) \geq m_{k}\left(P_{i} \cup T_{s_{1}, d_{1}}\right) \geq m_{k}\left(T_{d+1, d-2}\right)$.

Now suppose that $s_{1}=d_{1}+2$. In this case, $G_{1}$ is obtained by attaching respectively paths $P_{l}\left(0 \leq l \leq d_{1}-2\right)$ and $P_{d_{1}-l-2}$ to the two non-adjacent vertices in $K_{4}-e$. If $d_{1}=2$, then $i \geq d-3$. It can be easily checked that $m_{k}\left(K_{4}-e\right) \geq m_{k}\left(T_{5,2}\right)$. Thus $m_{k}(H) \geq m_{k}\left(P_{i} \cup\left(K_{4}-e\right)\right) \geq m_{k}\left(P_{i} \cup T_{5,2}\right) \geq$ $m_{k}\left(T_{d+1, d-2}\right)$. If $d_{1} \geq 3$, choose an edge $u_{0} u_{1}$ of $G_{1}$ such that $u_{0}$ and $u_{1}$ are both of degree 3 in $K_{4}-e$. Then we get

$$
\begin{aligned}
m_{k}(H) & \geq m_{k}\left(P_{i} \cup G_{1}\right) \\
& =m_{k}\left(P_{i} \cup G_{1}-u_{0} u_{1}\right)+m_{k-1}\left(P_{i} \cup G_{1}-u_{0}-u_{1}\right) \\
& \geq m_{k}\left(P_{i} \cup U_{s_{1}, d_{1}}\right)+m_{k-1}\left(P_{i} \cup P_{l+1} \cup P_{d_{1}-l-1}\right) \\
& \geq m_{k}\left(P_{i} \cup T_{s_{1}, d_{1}}\right)+m_{k-1}\left(P_{i} \cup P_{d_{1}-1}\right) \\
& \geq m_{k}\left(P_{i} \cup T_{d-i+1, d-i-1}\right)+m_{k-1}\left(P_{i} \cup P_{d-i-2}\right) \\
& \geq m_{k}\left(T_{d, d-2}\right)+m_{k-1}\left(P_{d-3}\right) \\
& =m_{k}\left(T_{d+1, d-2}\right) .
\end{aligned}
$$

Next, suppose that $v$ lies outside $P(G)$.

In this case, $H \supseteq G_{2}$ or $H \supseteq C_{a} \cdot C_{b} \cup P(G)$, where $G_{2} \in \mathcal{B}(s, d)$ with $d+2 \leq s \leq n-2$. It is easy to verify that $m_{k}(H) \geq m_{k}\left(T_{d+1, d-2}\right)$.

Case 3 If any diametrical path of $G$ contains all pendent vertices in $G$.

We can obtain that $G \succ B_{n, d}$ by similar arguments as those in Case 3 of Theorem 2.

Consequently, the proof is complete.

Combining Theorems 4.4 and 4.5, we obtain the following main result of this section. 
Theorem 4.6 Let $G \in \mathcal{B}(n, d)$ with $n \geq 8,3 \leq d \leq n-3$ and $G \neq B_{n, d}$. Then $M E(G)>M E\left(B_{n, d}\right)$.

Proof. According to Theorems 4.4 and 4.5, we have known that $G \succ B_{n, d}$. And then using the increasing property (namely, $G_{1} \succ G_{2} \Longrightarrow M E\left(G_{1}\right)>$ $\left.M E\left(G_{2}\right)\right)$, we get the result we want.

We will conclude this section by discussing the case $d=n-2$. Since any graph $G$ in $\mathcal{B}(n, n-2)$ is of the form $B_{n}^{s}$ (as shown in Figure 9), where $0 \leq s \leq\lfloor n / 2\rfloor-2$. Through simple analysis, we get the following result.

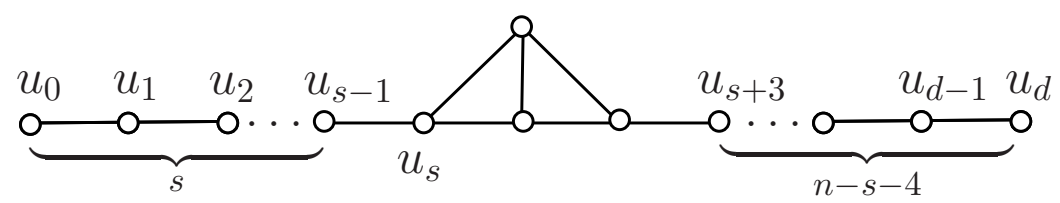

Figure 9: Graph $B_{n}^{s}$ with $0 \leq s \leq\lfloor n / 2\rfloor-2$.

Theorem 4.7 Let $G \in \mathcal{B}(n, n-2)$ with $n \geq 6$ and $G \neq B_{n}^{1}$, then $M E(G)>$ $M E\left(B_{n}^{1}\right)$.

Proof. Since $G \in \mathcal{B}(n, n-2)$ and $G \neq B_{n}^{1}$, then $G$ is $B_{n}^{s}$, where $s=$ $0,2, \ldots,\lfloor n / 2\rfloor-2$.

Case $1 G=B_{n}^{0}$.

Then $G-u_{d}=B_{n}^{0}-u_{d}=B_{n}^{1}-u_{0}$ and $G-u_{d-1}-u_{d}=B_{n}^{0}-u_{d-1}-u_{d} \succ$ $B_{n}^{1}-u_{0}-u_{1}$. Thus we have $G=B_{n}^{0} \succ B_{n}^{1}$ by Lemma 2.8,

Case $2 G=B_{n}^{s}(s=2, \ldots,\lfloor n / 2\rfloor-2)$.

Then

$$
\begin{aligned}
m_{k}\left(G=B_{n}^{s}\right) & =m_{k}\left(B_{n}^{s}-u_{s-2} u_{s-1}\right)+m_{k-1}\left(B_{n}^{s}-u_{s-2}-u_{s-1}\right) \\
& =m_{k}\left(P_{s-1} \cup B_{n-s+1}^{1}\right)+m_{k-1}\left(P_{s-2} \cup B_{n-s}^{0}\right),
\end{aligned}
$$

together with

$$
\begin{aligned}
m_{k}\left(B_{n}^{1}\right) & =m_{k}\left(B_{n}^{1}-u_{d-s+1} u_{d-s+2}\right)+m_{k-1}\left(B_{n}^{1}-u_{d-s+1}-u_{d-s+2}\right) \\
& =m_{k}\left(P_{s-1} \cup B_{n-s+1}^{1}\right)+m_{k-1}\left(P_{s-2} \cup B_{n-s}^{1}\right) .
\end{aligned}
$$

By Case 1, we have got that $B_{n-s}^{0} \succ B_{n-s}^{1}$, then $m_{k-1}\left(P_{s-2} \cup B_{n-s}^{0}\right) \geq$ $m_{k-1}\left(P_{s-2} \cup B_{n-s}^{1}\right)$ and $m_{2}\left(P_{s-2} \cup B_{n-s}^{0}\right)>m_{2}\left(P_{s-2} \cup B_{n-s}^{1}\right)$. Thus $G=$ $B_{n}^{s} \succ B_{n}^{1}$.

Therefore, we always have $G \succ B_{n}^{1}$. And then $M E(G)>M E\left(B_{n}^{1}\right)$. 


\section{Summary}

In [25, 35, the authors introduced the concept of "set-complexity", based on a context-dependent measure of information, and used this concept to describe the complexity of gene interaction networks. The binary graphs and edge-colored graphs are studied and the relation between complexity and structure of these graphs is examined in detail. In contrast, we put the emphasis on analyzing properties of spectra-based entropies and study interrelations thereof.

In this paper, we characterize the graphs with minimal matching energy among all unicyclic and bicyclic graphs with a given diameter $d$. With respect to matching energy of graphs, $U_{n, d}$ and $B_{n, d}$ are two extremal graphs in $\mathcal{U}(n, d)$ and $\mathcal{B}(n, d)$ respectively. Moreover, both of them are interesting and have the similar extremum property in other aspects. For example, among all unicyclic graphs of a given diameter, $U_{n, d}$ is the extremal graph on graph energy [29]. Besides, it is also the underling graph of the extremal graph on skew energy [38. In addition, $B_{n, d}$ has the minimal energy in one class of bicyclic graphs with a given diameter [39]. From this point, we guess that this two graphs may also be the extremal graphs on some other parameters of graphs. Studying the properties of this two graphs will be one of the future work of us. An important question is how general the bounds are. Obviously, the proof techniques use structural properties of the graphs under consideration and it may be intricate to extend the techniques when using more general graphs. On the other hand, the roots of graph polynomials could be used to characterize graphs structurally. This will be one of the future work. For more results, we refer to [11, 28].

Acknowledgement. The authors would like to thank the referees for valuable comments. The authors are supported by NSFC, PCSIRT, China Postdoctoral Science Foundation (2014M551015) and China Scholarship Council.

\section{References}

[1] Aihara, J. A new definition of Dewar-type resonance energies. J. Am. Chem. Soc. 1976, 98, 2750-2758.

[2] Bondy, J.A.; Murty, U.S.R. Graph Theory. Springer: Berlin, 2008.

[3] Bozkurt, S.B.; Bozkurt, D. Sharp upper bounds for energy and Randić energy. MATCH Commun. Math. Comput. Chem. 2013, 70, 669-680.

[4] Bozkurt, S.B.; Bozkurt, D. On incidence energy. MATCH Commun. Math. Comput. Chem. 2014, 72, 215-225. 
[5] Bozkurt, S.B.; Gutman, I. Estimating the incidence energy. MATCH Commun. Math. Comput. Chem. 2013, 70, 143-156.

[6] Chen, L.; Shi, Y. The maximal matching energy of tricyclic graphs. MATCH Commun. Math. Comput. Chem., in press.

[7] Das, K.C.; Gutman, I.; Cevik, A.S.; Zhou, B. On Laplacian energy. MATCH Commun. Math. Comput. Chem. 2013, 70, 689-696.

[8] Das, K.C.; Mojallal, S.A. Upper bounds for the energy of graphs. MATCH Commun. Math. Comput. Chem. 2013, 70, 657-662.

[9] Das, K.C.; Mojallal, S.A.; Gutman, I. Improving McClelland's lower bound for energy. MATCH Commun. Math. Comput. Chem. 2013, 70, 663-668.

[10] Das, K.C.; Sorgun, S. On Randić energy of graphs. MATCH Commun. Math. Comput. Chem. 2014, 72, 227-238.

[11] Dehmer, M.; Emmert-Streib, F.; Grabner M. A computational approach to construct a multivariate complete graph invariant. Information Sciences 2014, 260, 200-208.

[12] Dehmer, M.; Li, X.; Shi, Y. Connections between generalized graph entropies and graph energy. Complexity, in press, DOI: 10.1002/cplx.21539.

[13] Farrell, E.J. An introduction to matching polynomials. J. Comb. Theory B 1979, 27, 75-86.

[14] Ghorbani, M.; Faghani, M.; Ashrafi, A.R.; Heidari Rad, S.; Graovac, A. An upper bound for energy of matrices associated to an infinite class of fullerenes. MATCH Commun. Math. Comput. Chem. 2014, 71, 341-354.

[15] Gutman, I. The energy of a graph. Ber. Math.-Statist. Sekt. Forsch. Graz 1978, 103, 1-22.

[16] Gutman, I. The energy of a graph: old and new results. In: Algebraic Combinatorics and Applications, Betten, A.; Kohnert, A.; Laue, R.; Wassermann A., Eds., Springer: Berlin, 2001, pp. 196-211.

[17] Gutman, I.; Li, X.; Zhang, J. Graph energy. In: Analysis of Complex Networks: From Biology to Linguistics, Dehmer, M.; Emmert-Streb F., Eds., Wiley-VCH Verlag: Weinheim, 2009, pp. 145-174.

[18] Gutman, I.; Milun, M.; Trinajstić, N. Topological definition of delocalisation energy. MATCH Commun. Math. Comput. Chem. 1975, 1, 171-175. 
[19] Gutman, I. Acylclic systems with extremal Hückel $\pi$-electron energy. Theor. Chim. Acta 1977, 45, 79-87.

[20] Gutman, I.; Milun, M.; Trinajstić, N. Graph theory and molecular orbitals 19. Nonparametric resonance energies of arbitrary conjugated systems. J. Am. Chem. Soc. 1977, 99, 1692-1704.

[21] Gutman, I. The matching polynomial. MATCH Commun. Math. Comput. Chem. 1979, 6, 75-91.

[22] Gutman, I.; Polansky, O.E. Mathematical concepts in Organic Chemistry. Springer-Verlag: Berlin, 1986.

[23] Gutman, I.; Wagner, S. The matching energy of a graph. Discrete Appl. Match. 2012, 160, 2177-2187.

[24] Hou, Y. Unicyclic graphs with minimal energy. J. Math. Chem. 2001, 29, 163-168.

[25] Ignac, T., Sakhanenko, N., Galas, D. Complexity of networks II: The set complexity of edge-colored graphs, Complexity 2012, 17, 23-36.

[26] Ji, S.; Li, X.; Shi, Y. Extremal matching energy of bicyclic graphs. MATCH Commun. Math. Comput. Chem. 2013, 70, 697-706.

[27] Ji, S.; Ma, H. The extremal matching energy of graphs. Ars Combinatoria 2014, 115, 343-355.

[28] Kraus, V.; Dehmer, M.; Emmert-Streib, F. Probabilistic inequalities for evaluating structural network measures. Information Sciences, in Press, DOI: $10.1016 /$ j.ins.2014.07.018.

[29] Li, F.; Zhou, B. Minimal energy of unicyclic graphs of a given diameter. J. Math. Chem. 2008, 43, 476-484.

[30] Li, S.; Yan, W. The matching energy of graphs with given parameters. Discrete Appl. Math. 2014, 162, 415-420.

[31] Li, X.; Shi, Y.; Gutman, I. Graph Energy. Springer: New York, 2012.

[32] Li, X., Shi, Y., Wei, M., Li, J. On a conjecture about tricyclic graphs with maximal energy. MATCH Commun. Math. Comput. Chem. 2014, $72,183-214$.

[33] Mateljevic, M.; Bozin, V.; Gutman, I. Energy of a polynomial and the Coulson integral formula. J. Math. Chem. 2010, 48, 1062-1068.

[34] Milovanovic, I.Z.; Milovanovic, E.I.; Zakic, A. A short note on graph energy. MATCH Commun. Math. Comput. Chem. 2014, 72, 179-182. 
[35] Sakhanenko, N., Galas, D. Complexity of networks I: The setcomplexity of binary graphs, Complexity 2011, 17, 51-64.

[36] Stevanovic, D.; Milosevic, M.; Hic, P.; Pokorny, M. Proof of a conjecture on distance energy of complete multipartite graphs. MATCH Commun. Math. Comput. Chem. 2013, 70, 157-162.

[37] Yan, W.; Ye, L. On the minimal energy of trees with a given diameter. Appl. Math. Lett. 2005, 18, 1046-1052.

[38] Yang, X.; Gong, S.; Xu, G. Minimal skew energy of oriented unicyclic graphs with fixed diameter. J. Inequal. Appl. 2013, 418, 1-11.

[39] Yang, Y.; Zhou, B. Minimal energy of bicyclic graphs of a given diameter. MATCH Commun. Math. Comput. Chem. 2008, 59, 321-342.

[40] Zhou, B.; Li, F. On minimal energies of trees of a prescribed diameter. J. Math. Chem. 2006, 39, 465-473. 